\title{
ON COMPLEX LIE SUPERGROUPS AND SPLIT HOMOGENEOUS SUPERMANIFOLDS ${ }^{1}$
}

\author{
E.G. Vishnyakova
}

\begin{abstract}
It is well known that the category of real Lie supergroups is equivalent to the category of the so called (real) Harish-Chandra pairs, see $[3,6,7]$. That means that a Lie supergroup depends only on the underlying Lie group and its Lie superalgebra with certain compatibility conditions. More precisely, the structure sheaf of a Lie supergroup and the supergroup morphisms can be explicitly described in terms of the corresponding Lie superalgebra. In this paper, we give a proof of this result in the complexanalytic case. Furthermore, if $\left(G, \mathcal{O}_{G}\right)$ is a complex Lie supergroup and $H \subset G$ is a closed Lie subgroup, i.e. it is a Lie subsupergroup of $\left(G, \mathcal{O}_{G}\right)$ and its odd dimension is zero, we show that the corresponding homogeneous supermanifold $\left(G / H, \mathcal{O}_{G / H}\right)$ is split. In particular, any complex Lie supergroup is a split supermanifold.

It is well known that a complex homogeneous supermanifold may be nonsplit (see, e.g., [15]). We find here necessary and sufficient conditions for a complex homogeneous supermanifold to be split.
\end{abstract}

\section{Preliminaries}

We will use the word "supermanifold" in the sense of Berezin - Leites (see $[2,9]$ ). All the time, we will be interested in the real or complex-analytic version of the theory, denoting by $\mathbb{K}$ the ground field $\mathbb{R}$ or $\mathbb{C}$. Let $\left(M, \mathcal{O}_{M}\right)$ be a supermanifold. The underlying complex manifold $M$ is called the reduction of $\left(M, \mathcal{O}_{M}\right)$. We denote by $\mathcal{J}_{M} \subset \mathcal{O}_{M}$ the subsheaf of ideals generated by odd elements of the structure sheaf. The sheaf $\mathcal{O}_{M} / \mathcal{J}_{M}$ is naturally identified with the structure sheaf $\mathcal{F}_{M}$ of $M$. The natural homomorphism $\mathcal{O}_{M} \rightarrow \mathcal{F}_{M}$ will be denoted by $f \mapsto f_{\text {red }}$. A morphism $\phi:\left(M, \mathcal{O}_{M}\right) \rightarrow\left(N, \mathcal{O}_{N}\right)$ of supermanifolds will be denoted by $\phi=\left(\phi_{\text {red }}, \phi^{*}\right)$, where $\phi_{\text {red }}: M \rightarrow N$ is the corresponding mapping of the reductions and $\phi^{*}: \mathcal{O}_{N} \rightarrow\left(\phi_{\text {red }}\right)_{*}\left(\mathcal{O}_{M}\right)$ is the homomorphism of the structure sheaves. We denote by $\mathfrak{v}\left(M, \mathcal{O}_{M}\right)$ the Lie superalgebra of vector fields on $\left(M, \mathcal{O}_{M}\right)$. If $x \in M$ and $\mathfrak{m}_{x}$ is the maximal ideal of the local superalgebra $\left(\mathcal{O}_{M}\right)_{x}$, then the vector superspace $T_{x}\left(M, \mathcal{O}_{M}\right)=\left(\mathfrak{m}_{x} / \mathfrak{m}_{x}^{2}\right)^{*}$ is the tangent space to $\left(M, \mathcal{O}_{M}\right)$ at $x \in M$. From the inclusions $v\left(\mathfrak{m}_{x}\right) \subset\left(\mathcal{O}_{M}\right)_{x}$ and $v\left(\mathfrak{m}_{x}^{2}\right) \subset \mathfrak{m}_{x}$, where $v \in \mathfrak{v}\left(M, \mathcal{O}_{M}\right)$, it follows

\footnotetext{
${ }^{1}$ Work supported by SFB | TR 12 and by the Russian Foundation for Basic Research (grant no. 07-01-00230).
} 
that $v$ induces an even linear mapping $\operatorname{ev}_{x}(v): \mathfrak{m}_{x} / \mathfrak{m}_{x}^{2} \rightarrow\left(\mathcal{O}_{M}\right)_{x} / \mathfrak{m}_{x} \simeq \mathbb{C}$. In other words, $\operatorname{ev}_{x}(v) \in T_{x}\left(M, \mathcal{O}_{M}\right)$, and so we obtain an even linear map

$$
\mathrm{ev}_{x}: \mathfrak{v}\left(M, \mathcal{O}_{M}\right) \rightarrow T_{x}\left(M, \mathcal{O}_{M}\right)
$$

Let us take $Y_{x} \in T_{x}\left(M, \mathcal{O}_{M}\right)$. There is a neighborhood $\left(U, \mathcal{O}_{M}\right)$ of the point $x$ and a vector field $Y \in \mathfrak{v}\left(U, \mathcal{O}_{M}\right)$ such that $\operatorname{ev}_{x}(Y)=Y_{x}$. We may regard $Y_{x}$ as a linear function on $\left(\mathcal{O}_{M}\right)_{x}$. Namely, $Y_{x}\left(f_{x}\right):=\left(Y\left(f_{x}\right)\right)_{\text {red }}(x)$, where $f_{x} \in\left(\mathcal{O}_{M}\right)_{x}$. It is easy to verify that this definition doesn't depend on the choice of $Y$.

Let $\left(M, \mathcal{F}_{M}\right)$ be a complex-analytic, smooth or real-analytic manifold and let $\mathbf{E}_{M}$ be a (holomorphic, smooth or real-analytic) vector bundle over $M$. Denote by $\mathcal{E}_{M}$ the sheaf of (holomorphic, smooth or real-analytic) sections of $\mathbf{E}_{M}$. We get the supermanifold $\left(M, \bigwedge_{\mathcal{F}_{M}} \mathcal{E}_{M}\right)$ of the corresponding class. A supermanifold $\left(M, \mathcal{O}_{M}\right)$ is called split if $\mathcal{O}_{M} \simeq \bigwedge_{\mathcal{F}_{M}} \mathcal{E}_{M}$ for a certain vector bundle $\mathbf{E}_{M}$ of the corresponding class. It is known that any real (smooth or real analytic) supermanifold is split.

We may consider the supermanifold $(\mathrm{pt}, \mathbb{K})$ of dimension $(0 \mid 0)$, where pt is a point. If $\left(M, \mathcal{O}_{M}\right)$ is an arbitrary supermanifold then for any point $x \in M$ we denote by $\delta_{x}:(\mathrm{pt}, \mathbb{K}) \rightarrow\left(M, \mathcal{O}_{M}\right)$ the morphism, defined in the following way:

$$
\left(\delta_{x}\right)_{\mathrm{red}}(\mathrm{pt})=x, \quad \delta_{x}^{*}(f)= \begin{cases}f_{\mathrm{red}}(x), & \text { if } x \in U ; \\ 0, & \text { if } x \notin U,\end{cases}
$$

where $f \in \mathcal{O}_{M}(U)$ and $U \subset M$ is open.

Definition 1. A Lie supergroup is a group object in the category of supermanifolds, i.e., a supermanifold $\left(G, \mathcal{O}_{G}\right)$, for which the following three morphisms are defined: $\mu:\left(G, \mathcal{O}_{G}\right) \times\left(G, \mathcal{O}_{G}\right) \rightarrow\left(G, \mathcal{O}_{G}\right)$ (the multiplication morphism), $\iota:\left(G, \mathcal{O}_{G}\right) \rightarrow\left(G, \mathcal{O}_{G}\right)$ (the inversion morphism), $\varepsilon:(\mathrm{pt}, \mathbb{K}) \rightarrow\left(G, \mathcal{O}_{G}\right)$ (the identity morphism). Moreover, these morphisms should satisfy the usual conditions, modeling the group axioms:

1. $\mu \circ(\mu \times \mathrm{id})=\mu \circ(\mathrm{id} \times \mu)$;

2. $\mu \circ(\varepsilon \times \mathrm{id})=\mathrm{id}, \mu \circ(\mathrm{id} \times \varepsilon)=\mathrm{id}$;

3. $\mu \circ(\mathrm{id} \times \iota) \circ \operatorname{diag}=\varepsilon, \mu \circ(\iota \times \mathrm{id}) \circ \operatorname{diag}=\varepsilon$, where $\operatorname{diag}:\left(G, \mathcal{O}_{G}\right) \rightarrow$ $\left(G, \mathcal{O}_{G}\right) \times\left(G, \mathcal{O}_{G}\right)$ is the diagonal morphism.

The underlying manifold $G$ of a Lie supergroup is a (real or complex) Lie group. The element $e=\varepsilon_{\text {red }}(\mathrm{pt})$ is the identity element of $G$. Let $\left(G, \mathcal{O}_{G}\right)$, $\left(H, \mathcal{O}_{H}\right)$ be two Lie supergroups and $\mu_{G}, \mu_{H}$ the respective multiplication 
morphisms. A morphism $\Psi:\left(G, \mathcal{O}_{G}\right) \rightarrow\left(H, \mathcal{O}_{H}\right)$ is called a homomorphism of Lie supergroups if $\Psi \circ \mu_{G}=\mu_{H} \circ(\Psi \times \Psi)$. The corresponding mapping $\Psi_{\text {red }}: G \rightarrow H$ is a homomorphism of Lie groups.

Definition 2. An action of a Lie supergroup $\left(G, \mathcal{O}_{G}\right)$ on a supermanifold $\left(M, \mathcal{O}_{M}\right)$ is a morphism $\nu:\left(G, \mathcal{O}_{G}\right) \times\left(M, \mathcal{O}_{M}\right) \rightarrow\left(M, \mathcal{O}_{M}\right)$, such that the following conditions hold:

- $\nu \circ(\mu \times \mathrm{id})=\nu \circ(\mathrm{id} \times \nu)$;

- $\nu \circ(\varepsilon \times \mathrm{id})=\mathrm{id}$.

In this case $\nu_{\text {red }}$ is the action of $G$ on $M$.

We will denote by $\mathfrak{g}$ the Lie superalgebra of $\left(G, \mathcal{O}_{G}\right)$. By definition $\mathfrak{g}$ is the subalgebra of $\mathfrak{v}\left(G, \mathcal{O}_{G}\right)$, consisting of all right invariant vector fields on $\left(G, \mathcal{O}_{G}\right)$. (A vector field $Y$ on $\left(G, \mathcal{O}_{G}\right)$ is called right invariant if $(Y \otimes \mathrm{id}) \circ \mu^{*}=$ $\mu^{*} \circ Y$.) It is well known that any right invariant vector field $Y$ has the form

$$
Y=(X \otimes \mathrm{id}) \circ \mu^{*}
$$

for a certain $X \in T_{e}\left(G, \mathcal{O}_{G}\right)$ and the map $X \mapsto(X \otimes \mathrm{id}) \circ \mu^{*}$ is an isomorphism of the vector space $T_{e}\left(G, \mathcal{O}_{G}\right)$ onto $\mathfrak{g}$, see [20], Theorem 7.1.1. We will identify $\mathfrak{g}$ and $T_{e}\left(G, \mathcal{O}_{G}\right)$ using this isomorphism.

Let $\nu=\left(\nu_{\text {red }}, \nu^{*}\right):\left(G, \mathcal{O}_{G}\right) \times\left(M, \mathcal{O}_{M}\right) \rightarrow\left(M, \mathcal{O}_{M}\right)$ be an action. Then there is a homomorphism of the Lie superalgebras $\bar{\nu}: \mathfrak{g} \rightarrow \mathfrak{v}\left(M, \mathcal{O}_{M}\right)$, given by the formula

$$
X \mapsto(X \otimes \mathrm{id}) \circ \nu^{*} .
$$

As in [12], we use the following definition of a transitive action.

Definition 3. An action $\nu$ is called transitive if $\nu_{\text {red }}$ is transitive and the mapping $\mathrm{ev}_{x} \circ \bar{\nu}$ is surjective for all $x \in M$. (The map $\mathrm{ev}_{x}$ is given by (1).) In this case the supermanifold $\left(M, \mathcal{O}_{M}\right)$ is called $\left(G, \mathcal{O}_{G}\right)$-homogeneous. A supermanifold $\left(M, \mathcal{O}_{M}\right)$ is called homogeneous, if it possesses a transitive action of a certain Lie supergroup.

Let us consider the following compositions of the morphisms for any $g \in$ $G$ :

$$
\begin{aligned}
& l_{g}:\left(G, \mathcal{O}_{G}\right)=(g, \mathbb{K}) \times\left(G, \mathcal{O}_{G}\right) \stackrel{\delta_{g} \times \mathrm{id}}{\longrightarrow}\left(G, \mathcal{O}_{G}\right) \times\left(G, \mathcal{O}_{G}\right) \stackrel{\mu}{\rightarrow}\left(G, \mathcal{O}_{G}\right), \\
& r_{g}:\left(G, \mathcal{O}_{G}\right)=\left(G, \mathcal{O}_{G}\right) \times(g, \mathbb{K}) \stackrel{\mathrm{id} \times \delta_{g}}{\longrightarrow}\left(G, \mathcal{O}_{G}\right) \times\left(G, \mathcal{O}_{G}\right) \stackrel{\mu}{\rightarrow}\left(G, \mathcal{O}_{G}\right) .
\end{aligned}
$$

They are called the left and the right translation by $g$ respectively. Denote $\omega_{g}:=l_{g} \circ r_{g^{-1}}, g \in G$. The formula $\operatorname{Ad}_{G}(g):=\left(\mathrm{d} \omega_{g}\right)_{e}$ defines a representation $\operatorname{Ad}_{G}: G \rightarrow \operatorname{Aut}(\mathfrak{g})$, called the adjoint representation of the Lie group $G$ in $\mathfrak{g}$. 
Let $\left(M, \mathcal{O}_{M}\right)$ be a supermanifold. A subsupermanifold of $\left(M, \mathcal{O}_{M}\right)$ is a supermanifold $\left(N, \mathcal{O}_{N}\right)$ together with a morphism $\varphi:\left(N, \mathcal{O}_{N}\right) \rightarrow\left(M, \mathcal{O}_{M}\right)$ such that $\varphi_{\text {red }}: N \rightarrow M$ is a homeomorphism on the subset $\varphi_{\text {red }}(N) \subset M$ endowed with the induced topology and $(\mathrm{d} \varphi)_{p}$ is injective at every point $p \in M$. In this case we will sometimes use the notation $\left(M, \mathcal{O}_{M}\right) \subset\left(N, \mathcal{O}_{N}\right)$.

Let $\left(G, \mathcal{O}_{G}\right)$ be a Lie supergroup. We say that a subsupermanifold $\varphi$ : $\left(H, \mathcal{O}_{H}\right) \rightarrow\left(G, \mathcal{O}_{G}\right)$ is a Lie subsupergroup in $\left(G, \mathcal{O}_{G}\right)$ if $\left(H, \mathcal{O}_{H}\right)$ possesses a Lie supergroup structure, such that $\varphi$ is a homomorphism of the Lie supergroups. In this case we identify the Lie superalgebra $\mathfrak{h}$ of $\left(H, \mathcal{O}_{H}\right)$ with the Lie subsuperalgebra $(\mathrm{d} \varphi)_{e}(\mathfrak{h}) \subset \mathfrak{g}$.

Let us introduce the category of (super) Harish-Chandra pairs (see [3]). A Harish-Chandra pair is a pair $(G, \mathfrak{g})$ that consists of a Lie group $G$ and a Lie superalgebra $\mathfrak{g}=\mathfrak{g}_{\overline{0}} \oplus \mathfrak{g}_{\overline{1}}$, where $\mathfrak{g}_{\overline{0}}$ is the Lie algebra of $G$, provided with a representation $\alpha_{G}$ of $G$ in $\mathfrak{g}$ such that

- $\alpha_{G}$ preserves the parity and induces the adjoint representation of $G$ in $\mathfrak{g}_{\overline{0}}$,

- the differential $\left(\mathrm{d} \alpha_{G}\right)_{e}$ at the identity $e \in G$ coincides with the adjoint representation ad of $\mathfrak{g}_{0}$ in $\mathfrak{g}$.

Let $(G, \mathfrak{g})$ and $(H, \mathfrak{h})$ be two Harish-Chandra pairs. A morphism of $(G, \mathfrak{g})$ to $(H, \mathfrak{h})$ is a pair of homomorphisms $\Phi: G \rightarrow H, \varphi: \mathfrak{g} \rightarrow \mathfrak{h}$ with the following compatibility conditions:

- $(\mathrm{d} \Phi)_{e}=\left.\varphi\right|_{\mathfrak{g}_{\overline{0}}}$,

- $\varphi \circ \alpha_{G}(g)=\alpha_{H}(\Phi(g)) \circ \varphi$ for all $g \in G$.

It is clear how to associate a Harish-Chandra pair to a given Lie supergroup $\left(G, \mathcal{O}_{G}\right)$. Indeed, we may take the underlying Lie group $G$ with the Lie superalgebra $\mathfrak{g}$ of $\left(G, \mathcal{O}_{G}\right)$ equipped with the adjoint representation $\alpha_{G}=\operatorname{Ad}_{G}$. Furthermore, if $\Psi:\left(G, \mathcal{O}_{G}\right) \rightarrow\left(H, \mathcal{O}_{H}\right)$ is a homomorphism of Lie supergroups, then $\left(\Psi_{\text {red }},(\mathrm{d} \Psi)_{e}\right)$ is a morphism of the Harish-Chandra pairs $(G, \mathfrak{g}) \rightarrow(H, \mathfrak{h})$. This correspondence is a functor from the category of Lie supergroups to the category of Harish-Chandra pairs. From Theorem 3.5 and Remark 3.5.2 in [6] it can be deduced that this functor is an equivalence of categories in the real case. The proof in [6] uses the fact that $C^{\infty}$-supermanifold can be reconstructed from the algebra of global sections of its structure sheaf (see [6, Remark 2.14.2]). Since such reconstruction is in general impossible for holomorphic supermanifolds, this argument doesn't seem to immediately carry over to the holomorphic case. We will give a different proof of the equivalence that works both in the real and holomorphic cases. 
Let us denote the category of Harish-Chandra pairs by HCP and the category of Lie supergroups by SLG.

\section{Equivalence between HCP and SLG}

In this section we will prove that the categories HCP and SLG are equivalent. We denote by $\mathrm{Ob} C$ the set of objects of a category $C$ and by $\operatorname{Hom}(X, Y)$ the set of morphisms $X \rightarrow Y$ for two objects $X, Y \in \mathrm{Ob} C$. First, we shall describe a functor $F$ from the category HCP to SLG that was constructed by Koszul in [7]. Further, we show that for any object $Y \in$ Ob SLG there exists $X \in \mathrm{Ob}$ HCP such that $F(X)$ is isomorphic to $Y$. Finally, we prove that $F: \operatorname{Hom}(X, Y) \rightarrow \operatorname{Hom}(F(X), F(Y))$ is a bijection for every $X, Y \in$ Ob HCP. This will imply that $F$ determines an equivalence of our categories (see [19]).

2.1 The construction of $F$. If a (real or complex) Harish-Chandra pair $(G, \mathfrak{g})$ is given, then we can construct a Lie supergroup in the following way (see $[1,7])$. Let $\mathfrak{U}(\mathfrak{g})$ be the universal enveloping superalgebra of $\mathfrak{g}$ (see [18]). It is clear that $\mathfrak{U}(\mathfrak{g})$ is a $\mathfrak{U}\left(\mathfrak{g}_{\overline{0}}\right)$-module, where $\mathfrak{U}\left(\mathfrak{g}_{\overline{0}}\right)$ is the universal enveloping algebra of $\mathfrak{g}_{\overline{0}}$. The natural action of $\mathfrak{g}_{\overline{0}}$ on the sheaf $\mathcal{F}_{G}$ gives rise to a structure of $\mathfrak{U}\left(\mathfrak{g}_{\overline{0}}\right)$-module on $\mathcal{F}_{G}(U)$ for any open set $U \subset G$. Putting

$$
\widehat{\mathcal{O}}_{G}(U)=\operatorname{Hom}_{\mathfrak{U}\left(\mathfrak{g}_{\overline{0}}\right)}\left(\mathfrak{U}(\mathfrak{g}), \mathcal{F}_{G}(U)\right)
$$

for every open $U \subset G$, we get a sheaf $\widehat{\mathcal{O}}_{G}$ of $\mathbb{Z}_{2}$-graded vector spaces (here we assume that the functions from $\mathcal{F}_{G}(U)$ are even).

As a consequence of the graded version of Theorem of Poincaré-BirkhoffWitt, we obtain that $\mathfrak{U}\left(\mathfrak{g}_{\overline{0}}\right) \otimes \bigwedge\left(\mathfrak{g}_{\overline{1}}\right) \simeq \mathfrak{U}(\mathfrak{g})$ as $\mathfrak{U}\left(\mathfrak{g}_{\overline{0}}\right)$-modules (see $[7,18]$ ). The isomorphism is given by the formula $X \otimes Y \mapsto X \cdot \gamma(Y)$, where

$$
\gamma: \bigwedge\left(\mathfrak{g}_{\overline{1}}\right) \rightarrow \mathfrak{U}(\mathfrak{g}), \quad X_{1} \wedge \cdots \wedge X_{r} \mapsto \frac{1}{r !} \sum_{\sigma \in S_{r}}(-1)^{|\sigma|} X_{\sigma(1)} \cdots X_{\sigma(r)}
$$

The enveloping superalgebra $\mathfrak{U}(\mathfrak{g})$ has a Hopf superalgebra structure (see [18]). Indeed, the map

$$
\mathfrak{g} \rightarrow \mathfrak{U}(\mathfrak{g}) \otimes \mathfrak{U}(\mathfrak{g}), \quad X \mapsto X \otimes 1+1 \otimes X
$$

can be extended to a comultiplication map $\triangle: \mathfrak{U}(\mathfrak{g}) \rightarrow \mathfrak{U}(\mathfrak{g}) \otimes \mathfrak{U}(\mathfrak{g})$, and the antipode map $S: \mathfrak{U}(\mathfrak{g}) \rightarrow \mathfrak{U}(\mathfrak{g})$ is given by

$$
S(X)=-X, \quad S(1)=1, \quad S(Y \cdot Z)=(-1)^{p(Y) p(Z)} S(Z) \cdot S(Y),
$$


where $X \in \mathfrak{g}, Y, Z \in \mathfrak{U}(\mathfrak{g})$ and $p(V)$ is the parity of $V$. We can define a multiplication in each $\widehat{\mathcal{O}}_{G}(U)$, where $U \subset G$ is open, by

$$
f_{1} \cdot f_{2}:=\operatorname{Mult}_{\mathcal{F}_{G}} \circ\left(f_{1} \otimes f_{2}\right) \circ \triangle .
$$

Here $f_{1}, f_{2} \in \widehat{\mathcal{O}}_{G}(U)$ and by Mult $_{\mathcal{F}_{G}}$ is denoted the product in the sheaf $\mathcal{F}_{G}$. Note that for homogeneous $X, Y \in \mathfrak{U}(\mathfrak{g})$ and $f_{1}, f_{2} \in \widehat{\mathcal{O}}_{G}(U)$ we have

$$
\left(f_{1} \otimes f_{2}\right)(X \otimes Y)=(-1)^{p\left(f_{2}\right) p(X)} f_{1}(X) \otimes f_{2}(Y) .
$$

Furthermore, $\mathfrak{U}(\mathfrak{g})$ is super-cocommutative, i.e., $T^{s} \circ \Delta=\triangle$, where

$$
T^{s}(X \otimes Y)=(-1)^{p(X) p(Y)} Y \otimes X .
$$

Using (5) and (6) we get $f_{1} \cdot f_{2}=(-1)^{p\left(f_{1}\right) p\left(f_{2}\right)} f_{2} \cdot f_{1}$. Hence, the sheaf $\widehat{\mathcal{O}}_{G}$ is a sheaf of commutative associative superalgebras with unit.

Further, $\bigwedge\left(\mathfrak{g}_{\overline{1}}\right)$ is also a cosuperalgebra with comultiplication defined by $\triangle_{\mathfrak{g}_{\overline{1}}}(X)=X \otimes 1+1 \otimes X, \triangle_{\mathfrak{g}_{\overline{1}}}\left(X_{1} \wedge \cdots \wedge X_{r}\right)=\triangle_{\mathfrak{g}_{\overline{1}}}\left(X_{1}\right) \wedge \cdots \wedge \triangle_{\mathfrak{g}_{\overline{1}}}\left(X_{r}\right)$, where $X, X_{i} \in \mathfrak{g}_{\overline{1}}$. As above, this permits to regard $\operatorname{Hom}\left(\bigwedge\left(\mathfrak{g}_{\overline{1}}\right), \mathcal{F}_{G}\right)$ as a sheaf of superalgebras which we may identify with the sheaf of superalgebras $\mathcal{F}_{G} \otimes \bigwedge\left(\mathfrak{g}_{1}^{*}\right)$. Moreover, the homomorphism $\gamma$ given by $(4)$ is a homomorphism of cosuperalgebras. It follows that the mapping $\widehat{\mathcal{O}}_{G} \rightarrow \operatorname{Hom}\left(\bigwedge\left(\mathfrak{g}_{1}\right), \mathcal{F}_{G}\right)$, given by $f \mapsto f \circ \gamma$, is an isomorphism of sheaves of superalgebras. Hence, $\widehat{\mathcal{O}}_{G} \simeq \mathcal{F}_{G} \otimes \bigwedge\left(\mathfrak{g}_{1}^{*}\right)$, and $\left(G, \widehat{\mathcal{O}}_{G}\right)$ is a supermanifold. Clearly, it is split and corresponds to the trivial bundle over $G$ with the fibre $\bigwedge\left(\mathfrak{g}_{1}^{*}\right)$.

Now we are able to define a structure of a Lie supergroup on $\left(G, \widehat{\mathcal{O}}_{G}\right)$. The following formulas define the multiplication morphism, the inversion morphism and the identity morphism respectively (see [1]):

$$
\begin{aligned}
\mu^{*}(f)(X \otimes Y)(g, h) & =f\left(X \cdot \alpha_{G}(g)(Y)\right)(g h) ; \\
\iota^{*}(f)(X)(g) & =f\left(\alpha_{G}\left(g^{-1}\right)(S(X))\right)\left(g^{-1}\right) ; \\
\varepsilon^{*}(f) & =f(1)(e) .
\end{aligned}
$$

Here $X, Y \in \mathfrak{U}(\mathfrak{g}), f \in \widehat{\mathcal{O}}_{G}, g, h \in G$, and we identify the enveloping superalgebra $\mathfrak{U}(\mathfrak{g} \oplus \mathfrak{g})$ with the tensor product $\mathfrak{U}(\mathfrak{g}) \otimes \mathfrak{U}(\mathfrak{g})$. The group axioms can be easily verified, using the Hopf (super)algebra axioms. Note that $\left(G, \widehat{\mathcal{O}}_{G}\right)$ corresponds to the Harish-Chandra pair $(G, \mathfrak{g})$ and $\alpha_{G}=\operatorname{Ad}_{G}$.

Let $(\Phi, \varphi)$ be a morphism of Harish-Chandra pairs $(G, \mathfrak{g}) \rightarrow(H, \mathfrak{h})$. Then we can define a morphism $F((\Phi, \varphi))=\Psi:\left(G, \widehat{\mathcal{O}}_{G}\right) \rightarrow\left(H, \widehat{\mathcal{O}}_{H}\right)$ by the following formula:

$$
\Psi_{\text {red }}=\Phi, \quad \Psi^{*}(f)(X)(g)=f(\varphi(X))(\Phi(g)), \quad f \in \widehat{\mathcal{O}}_{H}, X \in \mathfrak{U}(\mathfrak{g}), g \in G .
$$


Let us prove that $\Psi$ is a homomorphism of Lie supergroups. We should show that $\Psi \circ \mu_{G}=\mu_{H} \circ(\Psi \times \Psi)$, where $\mu_{G}$ and $\mu_{H}$ are the multiplication morphisms of $\left(G, \widehat{\mathcal{O}}_{G}\right)$ and $\left(H, \widehat{\mathcal{O}}_{H}\right)$ respectively. By definition, we have

$$
\begin{gathered}
\mu_{G}^{*} \circ \Psi^{*}(f)(X \otimes Y)(g, h)=\Psi^{*}(f)\left(\alpha_{G}\left(h^{-1}\right)(X) \cdot Y\right)(g h)= \\
f\left(\varphi\left(\alpha_{G}\left(h^{-1}\right)(X) \cdot Y\right)\right)(\Phi(g h))
\end{gathered}
$$

and

$$
\begin{gathered}
\left(\Psi^{*} \times \Psi^{*}\right) \circ \mu_{H}^{*}(f)(X \otimes Y)(g, h)=\mu_{H}^{*}(f)(\varphi(X) \otimes \varphi(Y))(\Phi(g), \Phi(h))= \\
f\left(\alpha_{H}\left(\Phi\left(h^{-1}\right)\right)(\varphi(X)) \cdot \varphi(Y)\right)(\Phi(g) \Phi(h)) .
\end{gathered}
$$

Now our assertion follows from the definition of a morphism of HarishChandra pairs.

2.2 Isomorphisms of objects. Let $\left(G, \mathcal{O}_{G}\right)$ be a Lie supergroup and $\mathfrak{g}$ the corresponding Lie superalgebra. We want to prove that $\left(G, \mathcal{O}_{G}\right)$ is isomorphic to the Lie supergroup $F(P)$ which corresponds to the Harish-Chandra pair $P=(G, \mathfrak{g})$. Actually, we are going to prove a more general assertion, and therefore we first extend the functor $F$ to a wider class of objects.

Let $H$ be a closed Lie subgroup of $G$. As above, putting

$$
\widehat{\mathcal{O}}_{G / H}(U)=\operatorname{Hom}_{\mathfrak{U}\left(\mathfrak{g}_{\overline{0}}\right)}\left(\mathfrak{U}(\mathfrak{g}), \mathcal{F}_{G / H}(U)\right)
$$

for every open $U \subset G / H$, we get a sheaf of superalgebras $\widehat{\mathcal{O}}_{G / H}$. By the same argument as above, $\left(G / H, \widehat{\mathcal{O}}_{G / H}\right)$ is a split supermanifold and $\widehat{\mathcal{O}}_{G / H}$ is isomorphic to $\mathcal{F}_{G / H} \otimes \bigwedge\left(\mathfrak{g}_{1}^{*}\right)$ (see [7]). The isomorphism $\widehat{\mathcal{O}}_{G / H} \rightarrow$ $\operatorname{Hom}\left(\bigwedge\left(\mathfrak{g}_{\overline{1}}\right), \mathcal{F}_{G}\right) \simeq \mathcal{F}_{G / H} \otimes \bigwedge\left(\mathfrak{g}_{1}^{*}\right)$ is again given by the formula $f \mapsto f \circ \gamma$, where $\gamma$ is defined by $(4)$.

Further, let $\nu:\left(G, \mathcal{O}_{G}\right) \times\left(M, \mathcal{O}_{M}\right) \rightarrow\left(M, \mathcal{O}_{M}\right)$ be a transitive action. For simplicity we will denote the vector field $(X \otimes \mathrm{id}) \circ \nu^{*}$ also by $X$. Denote by $H$ the stabilizer of a certain point $x \in M$ by the action $\nu_{\text {red }}$. Our next aim is to define a morphism of supermanifolds $\left(G / H, \widehat{\mathcal{O}}_{G / H}\right) \rightarrow\left(M, \mathcal{O}_{M}\right)$. We will use the natural correspondence $X \mapsto \widehat{X}$ between even vector fields on $\left(M, \mathcal{O}_{M}\right)$ and vector fields on $M$ which is completely determined by the relation $\widehat{X}\left(f_{\text {red }}\right)=(X(f))_{\text {red }}$ for all $f \in \mathcal{O}_{M}$. Let $f \in \mathcal{O}_{M}(U)$, where $U$ is an open set in $M$. Denote by $\beta$ the natural biholomorphic mapping $G / H \rightarrow M$, $g H \mapsto g x$. Let us define the linear mapping $\Phi_{G / H}(f): \mathfrak{U}(\mathfrak{g}) \rightarrow \mathcal{F}_{G / H}\left(\beta^{-1}(U)\right)$ by

$$
\Phi_{G / H}(f)(X):=(-1)^{p(X) p(f)} \beta^{*}(X(f))_{\mathrm{red}},
$$

where $X \in \mathfrak{U}(\mathfrak{g})$ and $X$ and $f$ are homogeneous. If $X \in \mathfrak{g}_{\overline{0}}$, denote by $\bar{X}$ the corresponding vector field on $G / H$. Note that $\beta^{*} \circ \widehat{X}=\bar{X} \circ \beta^{*}$. The 
mapping $\Phi_{G / H}(f)$ is a homomorphism of $\mathfrak{U}\left(\mathfrak{g}_{\overline{0}}\right)$-modules. In fact, for any $X_{i} \in \mathfrak{g}_{\overline{0}}, Y_{j} \in \mathfrak{g}_{\overline{1}}$ we have

$$
\begin{gathered}
\Phi_{G / H}(f)\left(X_{1} \cdots X_{r} \cdot Y_{1} \cdots Y_{q}\right)=(-1)^{p\left(Y_{1} \cdots Y_{q}\right) p(f)} \beta^{*}\left(\left(X_{1} \cdots X_{r} \cdot Y_{1} \cdots Y_{q}\right)(f)\right)_{\mathrm{red}} \\
=(-1)^{p\left(Y_{1} \cdots Y_{q}\right) p(f)} \beta^{*}\left(\widehat{X}_{1} \cdots \widehat{X}_{r}\right)\left[\left(Y_{1} \cdots Y_{q}(f)\right)_{\mathrm{red}}\right]= \\
(-1)^{p\left(Y_{1} \cdots Y_{q}\right) p(f)}\left(\bar{X}_{1} \cdots \bar{X}_{r}\right) \beta^{*}\left[\left(Y_{1} \cdots Y_{q}(f)\right)_{\mathrm{red}}\right]= \\
\left(\bar{X}_{1} \cdots \bar{X}_{r}\right)\left(\Phi_{G / H}(f)\left(Y_{1} \cdots Y_{q}\right)\right)
\end{gathered}
$$

Proposition 1. $\Phi_{G / H}: \mathcal{O}_{M} \rightarrow \widehat{\mathcal{O}}_{G / H}$ is a homomorphism of sheaves of superalgebras and $\left(\beta, \Phi_{G / H}\right):\left(G, \widehat{\mathcal{O}}_{G / H}\right) \rightarrow\left(M, \mathcal{O}_{M}\right)$ is a morphism of supermanifolds.

To prove Proposition 1, we need the following two lemmas.

Lemma 1. Let $X_{1}, \ldots, X_{r} \in \mathfrak{g}_{\overline{1}}$, then

$$
\triangle\left(X_{1} \cdots X_{r}\right)=\sum_{a+b=r}(-1)^{|\tau|} X_{k_{1}} \cdots X_{k_{a}} \otimes X_{l_{1}} \cdots X_{l_{b}}
$$

where $k_{1}<\cdots<k_{a}, l_{1}<\cdots<l_{b}$ and $\tau$ is the permutation such that

$$
\tau\left(k_{1}, \ldots, k_{a}, l_{1}, \ldots, l_{b}\right)=(1, \ldots, r) .
$$

Proof. For $r=1$ the formula is just the definition of $\triangle$. Further, using induction, we get

$$
\begin{gathered}
\triangle\left(X_{1} \cdots X_{r+1}\right)=\triangle\left(X_{1} \cdots X_{r}\right) \cdot \triangle\left(X_{r+1}\right)= \\
\left(\sum_{a+b=r}(-1)^{|\tau|} X_{k_{1}} \cdots X_{k_{a}} \otimes X_{l_{1}} \cdots X_{l_{b}}\right) \cdot\left(X_{r+1} \otimes 1+1 \otimes X_{r+1}\right) \\
=\sum_{a+b=r}(-1)^{|\tau|+b} X_{k_{1}} \cdots X_{k_{a}} \cdot X_{r+1} \otimes X_{l_{1}} \cdots X_{l_{b}}+ \\
\sum_{a+b=r}(-1)^{|\tau|} X_{k_{1}} \cdots X_{k_{a}} \otimes X_{l_{1}} \cdots X_{l_{b}} \cdot X_{r+1}= \\
\sum_{a^{\prime}+b^{\prime}=r+1}(-1)^{\left|\tau^{\prime}\right|} X_{k_{1}} \cdots X_{k_{a^{\prime}}} \otimes X_{l_{1}} \cdots X_{l_{b^{\prime}}},
\end{gathered}
$$

where $k_{1}<\cdots<k_{a^{\prime}}, l_{1}<\cdots<l_{b^{\prime}}$ and

$$
\tau^{\prime}\left(k_{1}, \ldots, k_{a}^{\prime}, l_{1}, \ldots, l_{b}^{\prime}\right)=(1, \ldots, r+1) . \square
$$

Lemma 2. Let $X_{1}, \ldots, X_{r} \in \mathfrak{g}_{\overline{1}}$ and $f_{1}, f_{2} \in \mathcal{O}_{M}$, then

$$
\left(X_{1} \cdots X_{r}\right)\left(f_{1} f_{2}\right)=\sum_{a+b=r}(-1)^{|\tau|+p\left(f_{1}\right) b}\left(X_{k_{1}} \cdots X_{k_{a}}\right)\left(f_{1}\right)\left(X_{l_{1}} \cdots X_{l_{b}}\right)\left(f_{2}\right),
$$


where $k_{1}<\cdots<k_{a}, l_{1}<\cdots<l_{b}$ and $\tau\left(k_{1}, \ldots, k_{a}, l_{1}, \ldots, l_{b}\right)=(1, \ldots, r)$.

Proof. For $r=1$ the formula is simply the Leibniz rule. Again, using induction, we get

$$
\begin{gathered}
\left(X_{1} \cdots X_{r+1}\right)\left(f_{1} f_{2}\right)= \\
X_{1}\left(\sum_{a+b=r}(-1)^{|\tau|+p\left(f_{1}\right) b}\left(X_{k_{1}} \cdots X_{k_{a}}\right)\left(f_{1}\right)\left(X_{l_{1}} \cdots X_{l_{b}}\right)\left(f_{2}\right)\right)= \\
\sum_{a+b=r}(-1)^{|\tau|+p\left(f_{1}\right) b}\left(X_{1} \cdot X_{k_{1}} \cdots X_{k_{a}}\right)\left(f_{1}\right)\left(X_{l_{1}} \cdots X_{l_{b}}\right)\left(f_{2}\right)+ \\
(-1)^{|\tau|+p\left(f_{1}\right) b+p\left(f_{1}\right)+a}\left(X_{k_{1}} \cdots X_{k_{a}}\right)\left(f_{1}\right)\left(X_{1} \cdot X_{l_{1}} \cdots X_{l_{b}}\right)\left(f_{2}\right)= \\
\sum_{a^{\prime}+b^{\prime}=r+1}(-1)^{\left|\tau^{\prime}\right|+p\left(f_{1}\right) b^{\prime}}\left(X_{k_{1}} \cdots X_{k_{a^{\prime}}}\right)\left(f_{1}\right)\left(X_{l_{1}} \cdots X_{l_{b^{\prime}}}\right)\left(f_{2}\right),
\end{gathered}
$$

where $k_{1}<\cdots<k_{a^{\prime}}, l_{1}<\cdots<l_{b^{\prime}}$ and

$$
\tau^{\prime}\left(k_{1}, \ldots, k_{a}^{\prime}, l_{1}, \ldots, l_{b}^{\prime}\right)=(1, \ldots, r+1) .
$$

Proof of Proposition 1. We should check the equality

$$
\left(\Phi_{G / H}\left(f_{1}\right) \cdot \Phi_{G / H}\left(f_{2}\right)\right)(X)=\left(\Phi_{G / H}\left(f_{1} f_{2}\right)\right)(X)
$$

for $X \in \mathfrak{U}(\mathfrak{g}), f_{1}, f_{2} \in \mathcal{O}_{M}$. Without loss of generality we may assume that $X=X_{1} \cdots X_{r}, X_{i} \in \mathfrak{g}_{\overline{1}}$, and that $f_{1}, f_{2}$ are homogeneous. Using (10), we get

$$
\begin{gathered}
\left(\Phi_{G / H}\left(f_{1}\right) \cdot \Phi_{G / H}\left(f_{2}\right)\right)\left(X_{1} \cdots X_{r}\right)= \\
\operatorname{Mult}_{\mathcal{F}_{G}}\left(\Phi_{G / H}\left(f_{1}\right) \otimes \Phi_{G / H}\left(f_{2}\right)\right)\left(\sum_{a+b=r}(-1)^{|\tau|} X_{k_{1}} \cdots X_{k_{a}} \otimes X_{l_{1}} \cdots X_{l_{b}}\right)= \\
\sum_{a+b=r}(-1)^{|\tau|+p\left(f_{2}\right) a} \Phi_{G / H}\left(f_{1}\right)\left(X_{k_{1}} \cdots X_{k_{a}}\right) \Phi_{G / H}\left(f_{2}\right)\left(X_{l_{1}} \cdots X_{l_{b}}\right)= \\
\sum_{a+b=r}(-1)^{|\tau|+p\left(f_{2}\right) a}(-1)^{p\left(f_{1}\right) a+p\left(f_{2}\right) b} \beta^{*}\left[\left(X_{k_{1}} \cdots X_{k_{a}}\right)\left(f_{1}\right)\left(X_{l_{1}} \cdots X_{l_{b}}\right)\left(f_{2}\right)\right]_{\mathrm{red}} \\
\sum_{a+b=r}(-1)^{|\tau|+p\left(f_{2}\right) r+p\left(f_{1}\right) a} \beta^{*}\left[\left(X_{k_{1}} \cdots X_{k_{a}}\right)\left(f_{1}\right)\left(X_{l_{1}} \cdots X_{l_{b}}\right)\left(f_{2}\right)\right]_{\mathrm{red}} .
\end{gathered}
$$

On the other hand, by (11) we have

$$
\begin{aligned}
& \left(\Phi_{G / H}\left(f_{1} f_{2}\right)\right)\left(X_{1} \cdots X_{r}\right)=(-1)^{r\left(p\left(f_{1}\right)+p\left(f_{2}\right)\right)} \beta^{*}\left(\left(X_{1} \cdots X_{r}\right)\left(f_{1} f_{2}\right)\right)_{\mathrm{red}}= \\
& (-1)^{r\left(p\left(f_{1}\right)+p\left(f_{2}\right)\right)} \sum_{a+b=r}(-1)^{|\tau|+p\left(f_{1}\right) b} \beta^{*}\left[\left(X_{k_{1}} \cdots X_{k_{a}}\right)\left(f_{1}\right)\left(X_{l_{1}} \cdots X_{l_{b}}\right)\left(f_{2}\right)\right]_{\mathrm{red}}= \\
& \sum_{a+b=r}(-1)^{|\tau|+p\left(f_{2}\right) r+p\left(f_{1}\right) a} \beta^{*}\left[\left(X_{k_{1}} \cdots X_{k_{a}}\right)\left(f_{1}\right)\left(X_{l_{1}} \cdots X_{l_{b}}\right)\left(f_{2}\right)\right]_{\mathrm{red}} .
\end{aligned}
$$

The equality proves the first assertion of Proposition 1. The second assertion follows from the first one. The proof is complete. 
We may consider the special case when the odd dimension of $\left(M, \mathcal{O}_{M}\right)$ is equal to the odd dimension of $\left(G, \mathcal{O}_{G}\right)$. Later we will see that this condition is equivalent to the following one:

$$
\operatorname{dim}\left(H, \mathcal{O}_{H}\right)=\operatorname{dim} H \mid 0,
$$

where $\left(H, \mathcal{O}_{H}\right)$ is the stabilizer of $x$ (see below). In other words, $\left(H, \mathcal{O}_{H}\right)=$ $\left(H, \mathcal{F}_{H}\right)$ is an ordinary Lie subgroup of $G=\left(G, \mathcal{F}_{G}\right)$.

Proposition 2. The morphism $\left(\beta, \Phi_{G / H}\right):\left(G / H, \widehat{\mathcal{O}}_{G / H}\right) \rightarrow\left(M, \mathcal{O}_{M}\right)$ is a submersion. If in addition $\operatorname{dim}\left(M, \mathcal{O}_{M}\right)=\operatorname{dim} M \mid \operatorname{dim} \mathfrak{g}_{\overline{1}}$, then $\left(\beta, \Phi_{G / H}\right)$ is an isomorphism of supermanifolds. In particular, all complex homogeneous supermanifolds of this kind are split.

Proof. Let $y \in M$. Denote by $\mathfrak{m}_{y}$ and $\widehat{\mathfrak{m}}_{y}$ the maximal ideals of the local superalgebras $\left(\mathcal{O}_{M}\right)_{y}$ and $\left(\widehat{\mathcal{O}}_{G / H}\right)_{y}$ respectively. It is easy to see that

$$
\begin{gathered}
\widehat{\mathfrak{m}}_{y}=\left\{h \in\left(\widehat{\mathcal{O}}_{G / H}\right)_{y} \mid h(1)(y)=0\right\}, \\
\widehat{\mathfrak{m}}_{y}^{2}=\left\{h \in \widehat{\mathfrak{m}}_{y} \mid h(X)(y)=0 \text { for all } X \in \mathfrak{g}\right\} .
\end{gathered}
$$

Note that $\Phi_{G / H}\left(\mathfrak{m}_{y}\right) \subset \widehat{\mathfrak{m}}_{y}$. Let us take $f \in \mathfrak{m}_{y} \backslash \mathfrak{m}_{y}^{2}$. The action $\nu$ is transitive, hence there exists $X \in \mathfrak{g}$ such that $(X(f))_{\text {red }}(y) \neq 0$. Therefore, $\Phi_{G / H}(f)(X)(y) \neq 0$ and $\Phi_{G / H}(f) \in \widehat{\mathfrak{m}}_{y} \backslash \widehat{\mathfrak{m}}_{y}^{2}$. It follows that the induced map $\mathfrak{m}_{y} / \mathfrak{m}_{y}^{2} \rightarrow \widehat{\mathfrak{m}}_{y} / \widehat{\mathfrak{m}}_{y}^{2}$ is injective. Hence, the dual map $\left(\widehat{\mathfrak{m}}_{y} / \widehat{\mathfrak{m}}_{y}^{2}\right)^{*} \rightarrow\left(\mathfrak{m}_{y} / \mathfrak{m}_{y}^{2}\right)^{*}$ (or the differential of $\left(\beta, \Phi_{G / H}\right)$ at the point $\left.y\right)$ is surjective. Hence $\left(\beta, \Phi_{G / H}\right)$ is a submersion. Further, since

$$
\operatorname{dim}\left(M, \mathcal{O}_{M}\right)=\operatorname{dim}\left(G / H, \widehat{\mathcal{O}}_{G / H}\right)=\operatorname{dim} M \mid \operatorname{dim} \mathfrak{g}_{\overline{1}},
$$

we get that the differential is an isomorphism at every point $y \in M$. Hence, $\left(\beta, \Phi_{G / H}\right)$ is a local isomorphism (see [9], Inverse Function Theorem). But the mapping $\beta$ is bijective, hence $\left(\beta, \Phi_{G / H}\right)$ is an isomorphism.

In the case when $\left(M, \mathcal{O}_{M}\right)=\left(G, \mathcal{O}_{G}\right)$ and $\nu=\mu$ we get

Corollary. All complex supergroups are split supermanifolds.

This fact also follows from the results of [11]. Note that not all complex homogeneous supermanifolds are split. Some examples can be found in [15].

Now we return to the correspondence between Lie supergroups and HarishChandra pairs. In the case when $\left(M, \mathcal{O}_{M}\right)=\left(G, \mathcal{O}_{G}\right)$ formula (9) defines a homomorphism of sheaves of superalgebras $\Phi_{G}: \mathcal{O}_{G} \rightarrow \widehat{\mathcal{O}}_{G}$ if we put $x=e$ and $\beta=$ id. Define by $\Phi_{G} \times \Phi_{G}$ the second component of the morphism

$$
\left(\mathrm{id}, \Phi_{G}\right) \times\left(\mathrm{id}, \Phi_{G}\right):\left(G, \widehat{\mathcal{O}}_{G}\right) \times\left(G, \widehat{\mathcal{O}}_{G}\right) \rightarrow\left(G, \mathcal{O}_{G}\right) \times\left(G, \mathcal{O}_{G}\right) .
$$


Lemma 3. We have $\Phi_{G} \times \Phi_{G}=\Phi_{G \times G}$.

Proof. It is sufficient to check the equality

$$
\left.\left(\Phi_{G} \times \Phi_{G}\right)\right|_{\operatorname{pr}_{i}^{*}\left(\mathcal{O}_{G}\right)}=\left.\Phi_{G \times G}\right|_{\operatorname{pr}_{i}^{*}\left(\mathcal{O}_{G}\right)}, \quad i=1,2,
$$

where $\operatorname{pr}_{i}:\left(G, \mathcal{O}_{G}\right) \times\left(G, \mathcal{O}_{G}\right) \rightarrow\left(G, \mathcal{O}_{G}\right)$ is the projection onto the $i$-th factor. Let $\widehat{\mathrm{pr}}_{i}:\left(G, \widehat{\mathcal{O}}_{G}\right) \times\left(G, \widehat{\mathcal{O}}_{G}\right) \rightarrow\left(G, \widehat{\mathcal{O}}_{G}\right)$ be also the projection onto the $i$-th factor and $h \in \widehat{\mathcal{O}}_{G}$. For example $\widehat{\mathrm{pr}}_{1}^{*}(h)$ has the following form as a $\mathfrak{U}\left((\mathfrak{g} \oplus \mathfrak{g})_{\overline{0}}\right)$-module homomorphism of $\mathfrak{U}(\mathfrak{g} \oplus \mathfrak{g}) \simeq \mathfrak{U}(\mathfrak{g}) \otimes \mathfrak{U}(\mathfrak{g})$ to $\mathcal{F}_{G \times G}$ :

$$
\widehat{\operatorname{pr}}_{1}^{*}(h)\left(X^{r} \cdot Y^{q}\right)\left(g_{1}, g_{2}\right)= \begin{cases}0, & \text { if } q \neq 0 \\ h\left(X^{q}\right)\left(g_{1}\right), & \text { if } q=0\end{cases}
$$

Here $X^{r}:=X_{1} \cdots X_{r}, Y^{q}:=Y_{1} \cdots Y_{q}$, where $X_{i}$ are from the first copy of $\mathfrak{g}$ and $Y_{j}$ are from the second one.

Let us take $f \in\left(\mathcal{O}_{G}\right)_{\bar{i}}$. By definition of $\Phi_{G} \times \Phi_{G}$ and by (12) we get:

$$
\begin{aligned}
\left(\Phi_{G} \times \Phi_{G}\right)\left(\operatorname{pr}_{1}^{*}(f)\right)\left(X^{r} \cdot Y^{q}\right)\left(g_{1}, g_{2}\right) & = \\
\widehat{p r}_{1}^{*}\left(\Phi_{G}(f)\right)\left(X^{r} \cdot Y^{q}\right)\left(g_{1}, g_{2}\right) & = \begin{cases}0, & \text { if } q \neq 0 \\
\Phi_{G}(f)\left(X^{r}\right)\left(g_{1}\right), & \text { if } q=0\end{cases}
\end{aligned}
$$

On the other hand,

$$
\begin{aligned}
& \Phi_{G \times G}\left(\operatorname{pr}_{1}^{*}(f)\right)\left(X^{r} \cdot Y^{q}\right)\left(g_{1}, g_{2}\right)=(-1)^{p\left(X^{r} \cdot Y^{q}\right) p(f)}\left[X^{r} \cdot Y^{q}\left(\operatorname{pr}_{1}^{*}(f)\right)\right]_{\mathrm{red}}\left(g_{1}, g_{2}\right) \\
& = \begin{cases}0, & \text { if } q \neq 0 \\
(-1)^{p\left(X^{r}\right) p(f)}\left(X^{r}(f)\right)_{\mathrm{red}}\left(g_{1}\right), & \text { if } q=0 .\end{cases}
\end{aligned}
$$

This completes the proof.

Proposition 3. $\left(\mathrm{id}, \Phi_{G}\right):\left(G, \widehat{\mathcal{O}}_{G}\right) \rightarrow\left(G, \mathcal{O}_{G}\right)$ is an isomorphism of Lie supergroups.

Proof. Due to Lemma 3, we should check that $\left(\Phi_{G \times G} \circ \mu^{*}\right)(f)=\left((\hat{\mu})^{*} \circ \Phi_{G}\right)(f)$ for all $f \in \mathcal{O}_{G}$, where $\hat{\mu}$ is the multiplication morphism for $\left(G, \widehat{\mathcal{O}}_{G}\right)$. Let $X^{r}$ and $Y^{q}$ be as in the proof of Lemma 3. Recall that in Preliminaries the morphism $\delta_{x}:(\mathrm{pt}, \mathbb{K}) \rightarrow\left(M, \mathcal{O}_{M}\right)$ was defined. Obviously we have

$$
\begin{gathered}
\Phi_{G \times G}\left(\mu^{*}(f)\right)\left(X^{r} \cdot Y^{q}\right)\left(g_{1}, g_{2}\right)=(-1)^{p\left(X^{r} \cdot Y^{q}\right) p(f)}\left(\delta_{g_{1}}^{*} \otimes \delta_{g_{2}}^{*}\right) \circ X^{r} \cdot Y^{q} \circ \\
\mu^{*}(f)=(-1)^{p\left(X^{r} \cdot Y^{q}\right) p(f)}\left(\delta_{g_{1}}^{*} \circ X^{r} \otimes \delta_{g_{2}}^{*} \circ Y^{q}\right) \circ \mu^{*}(f), g_{1}, g_{2} \in G .
\end{gathered}
$$

We will use the following equalities:

$$
\begin{gathered}
\left(r_{g_{1}}^{*} \otimes \mathrm{id}\right) \circ \mu^{*}=\left(\mathrm{id} \otimes r_{g_{1}}^{*} \circ \omega_{g_{1}}^{*}\right) \circ \mu^{*}, \\
r_{g_{i}}^{*} \circ X=X \circ r_{g_{i}}^{*}, i=1,2 ; \\
\delta_{g_{2}}^{*} \circ r_{g_{1}}^{*}=\delta_{g_{2} g_{1}}^{*}, \quad \delta_{g_{2} g_{1}}^{*} \circ \omega_{g_{1}}^{*}=\delta_{g_{1} g_{2}}^{*} .
\end{gathered}
$$


Here $g_{1}, g_{2} \in G, X \in \mathfrak{U}(\mathfrak{g})$. By (14) we get

$$
\begin{aligned}
\left(\delta_{g_{1}}^{*} \circ X^{r} \otimes\right. & \left.\delta_{g_{2}}^{*} \circ Y^{q}\right) \circ \mu^{*}(f)=\left(\delta_{e}^{*} \circ r_{g_{1}}^{*} \circ X^{r} \otimes \delta_{g_{2}}^{*} \circ Y^{q}\right) \circ \mu^{*}(f)= \\
& \left(\delta_{e}^{*} \circ X^{r} \otimes \delta_{g_{2}}^{*} \circ Y^{q}\right) \circ\left(r_{g_{1}}^{*} \otimes \mathrm{id}\right) \circ \mu^{*}(f)= \\
& \left(\delta_{e}^{*} \circ X^{r} \otimes \delta_{g_{2}}^{*} \circ Y^{q} \circ r_{g_{1}}^{*} \circ \omega_{g_{1}}^{*}\right) \circ \mu^{*}(f)= \\
& \left(\delta_{e}^{*} \circ X^{r} \otimes \delta_{g_{2}}^{*} \circ r_{g_{1}}^{*} \circ Y^{q} \circ \omega_{g_{1}}^{*}\right) \circ \mu^{*}(f)= \\
& \left(\delta_{e}^{*} \circ X^{r} \otimes \delta_{g_{2} g_{1}}^{*} \circ Y^{q} \circ \omega_{g_{1}}^{*}\right) \circ \mu^{*}(f)= \\
& \left(\delta_{e}^{*} \circ X^{r} \otimes \delta_{g_{1} g_{2}}^{*} \circ \omega_{g_{1}^{-1}}^{*} \circ Y^{q} \circ \omega_{g_{1}}^{*}\right) \circ \mu^{*}(f)= \\
& \left(\delta_{e}^{*} \circ X^{r} \otimes \delta_{g_{1} g_{2}}^{*} \circ \operatorname{Ad}_{G}\left(g_{1}\right)\left(Y^{q}\right)\right) \circ \mu^{*}(f) .
\end{aligned}
$$

By induction it is easy to check that

$$
\begin{aligned}
& \left(Y^{q}\right)(f)=(-1)^{A\left(Y^{q}\right)}\left(\delta_{e}^{*} \circ Y_{q} \otimes \cdots \otimes \delta_{e}^{*} \circ Y_{1} \otimes \mathrm{id}\right) \circ\left(\mu^{q}\right)^{*}(f), \text { where } \\
& A\left(Y^{q}\right)=p\left(Y_{q-1}\right) p\left(Y_{q}\right)+p\left(Y_{q-2}\right) p\left(Y_{q-1} \cdot Y_{q}\right)+\cdots+p\left(Y_{1}\right) p\left(Y_{2} \cdots Y_{q}\right) .
\end{aligned}
$$

Here $\mu^{q}$ is the multiplication morphism of $q+1$ copies of $\left(G, \mathcal{O}_{G}\right)$. Indeed, for $q=1$ the assertion (16) is just the definition of a right invariant vector field. Further,

$$
\begin{gathered}
\left(Y^{q}\right)(f)=Y_{1}\left((-1)^{A\left(Y_{2} \cdots Y_{q}\right)}\left(\delta_{e}^{*} \circ Y_{q} \otimes \cdots \otimes \delta_{e}^{*} \circ Y_{2} \otimes \mathrm{id}\right)\left(\mu^{q-1}\right)^{*}(f)\right)= \\
(-1)^{A\left(Y_{2} \cdots Y_{q}\right)}(-1)^{p\left(Y_{1}\right) p\left(Y_{2} \cdots Y_{q}\right)}\left(\delta_{e}^{*} \circ Y_{q} \otimes \cdots \otimes \delta_{e}^{*} \circ Y_{1} \otimes \mathrm{id}\right)\left(\mu^{q}\right)^{*}(f)= \\
(-1)^{A\left(Y^{q}\right)}\left(\delta_{e}^{*} \circ Y_{q} \otimes \cdots \otimes \delta_{e}^{*} \circ Y_{1} \otimes \mathrm{id}\right)\left(\mu^{q}\right)^{*}(f) .
\end{gathered}
$$

By (16) we have

$$
\begin{aligned}
& \left(\delta_{e}^{*} \circ X^{r} \otimes \delta_{g_{1} g_{2}}^{*} \circ \operatorname{Ad}_{G}\left(g_{1}\right)\left(Y^{q}\right)\right) \circ \mu^{*}(f)=(-1)^{A\left(X^{r}\right)+A\left(Y^{q}\right)}\left(\delta_{e}^{*} \circ X_{r} \otimes \cdots\right. \\
& \left.\otimes \delta_{e}^{*} \circ X_{1} \otimes \delta_{e}^{*} \circ \operatorname{Ad}_{G}\left(g_{1}\right)\left(Y_{q}\right) \otimes \cdots \otimes \delta_{e}^{*} \circ \operatorname{Ad}_{G}\left(g_{1}\right)\left(Y_{1}\right) \otimes \delta_{g_{1} g_{2}}^{*}\right)\left(\mu^{r+q}\right)^{*}(f)= \\
& (-1)^{A\left(X^{r}\right)+A\left(Y^{q}\right)+A\left(Y^{q} \cdot X^{r}\right)}\left(\delta_{q_{1} g_{2}}^{*} \circ \operatorname{Ad}_{G}\left(g_{1}\right)\left(Y^{q}\right) \circ X^{r}\right)(f)= \\
& (-1)^{A\left(X^{r}\right)+A\left(Y^{q}\right)+A\left(Y^{q} \cdot X^{r}\right)}(-1)^{p\left(X^{r}\right) p\left(Y^{q}\right)}\left(\delta_{g_{1} g_{2}}^{*} \circ X^{r} \circ \operatorname{Ad}_{G}\left(g_{1}\right)\left(Y^{q}\right)\right)(f)= \\
& \left(\delta_{g_{1} g_{2}}^{*} \circ X^{r} \circ \operatorname{Ad}_{G}\left(g_{1}\right)\left(Y^{q}\right)\right)(f) .
\end{aligned}
$$

On the other hand,

$$
\begin{gathered}
\mu^{*}(\Phi(f))\left(X^{r} \cdot Y^{q}\right)\left(g_{1}, g_{2}\right)=\Phi(f)\left(X^{r} \cdot \operatorname{Ad}_{G}\left(g_{1}\right)\left(Y^{q}\right)\right)\left(g_{1} g_{2}\right)= \\
(-1)^{p\left(X^{r} \cdot Y^{q}\right) p(f)}\left(\delta_{g_{1} g_{2}}^{*} \circ X^{r} \cdot \operatorname{Ad}_{G}\left(g_{1}\right)\left(Y^{q}\right)\right)(f) .
\end{gathered}
$$

This completes the proof.

2.3 The bijection between morphisms. Let $(G, \mathfrak{g})$ and $(H, \mathfrak{h})$ be two HarishChandra pairs and $\left(G, \widehat{\mathcal{O}}_{G}\right)$ and $\left(H, \widehat{\mathcal{O}}_{H}\right)$ be the corresponding Lie supergroups with multiplication morphisms $\mu_{G}$ and $\mu_{H}$ respectively. Let $\Psi$ : $\left(G, \widehat{\mathcal{O}}_{G}\right) \rightarrow\left(H, \widehat{\mathcal{O}}_{H}\right)$ be a homomorphism of Lie supergroups. Let $X_{e} \in \mathfrak{g}$ 
and $X=\left(X_{e} \otimes \mathrm{id}\right) \circ \mu_{G}^{*}$ be the corresponding right invariant vector field on $\left(G, \widehat{\mathcal{O}}_{G}\right)$ and $Y=\left((\mathrm{d} \Psi)_{e} X_{e} \otimes \mathrm{id}\right) \circ \mu_{H}^{*}$. Then the vector fields $X$ and $Y$ are $\Psi$-related, i.e.

$$
X\left(\Psi^{*}(f)\right)=\Psi^{*}(Y(f)), \quad f \in \widehat{\mathcal{O}}_{H} .
$$

Now we are able to prove that $\Psi$ depends only on $\Psi_{\text {red }}$ and $(\mathrm{d} \Psi)_{e}$. Indeed,

$$
\begin{gathered}
{\left[\Psi^{*}(f)\left(X_{e}\right)\right](g)=(-1)^{p(X) p(f)}\left[X\left(\Psi^{*}(f)\right)\right]_{\mathrm{red}}(g)=} \\
(-1)^{p(X) p(f)}\left[\Psi^{*}(Y(f))\right]_{\mathrm{red}}(g)=(-1)^{p(X) p(f)}[Y(f)]_{\mathrm{red}}\left(\Psi_{\mathrm{red}}(g)\right)= \\
f\left((\mathrm{~d} \Psi)_{e} X_{e}\right)\left(\Psi_{\mathrm{red}}(g)\right),
\end{gathered}
$$

where $X \in \mathfrak{g}, f \in \widehat{\mathcal{O}}_{H}, g \in G$. It follows that all homomorphisms of $\left(G, \widehat{\mathcal{O}}_{G}\right)$ to $\left(H, \widehat{\mathcal{O}}_{H}\right)$ have the form (8) if we put $\varphi=(\mathrm{d} \Psi)_{e}, \Phi=\Psi_{\text {red }}$. Hence the map $F: \operatorname{Hom}(X, Y) \rightarrow \operatorname{Hom}(F(X), F(Y))$ is surjective. The injectivity of the map $F: \operatorname{Hom}(X, Y) \rightarrow \operatorname{Hom}(F(X), F(Y))$ is obvious.

2.4 The main result. We have proved the following theorem.

Theorem 1. The category of complex Lie supergroups is equivalent to the category of complex Harish-Chandra pairs.

Theorem 1 implies some important consequences: the existence of a Lie supergroup for a given Lie superalgebra, the existence of a Lie subsupergroup for a given Lie subsuperalgebra. (The last assertion we will discuss below.) Using Theorem 1, these two assertions can be proven in the complex-analytic case as in [6], Corollary to Theorem 3.7 and Theorem 3.8.

Remark. The same method can be used to prove Theorem 1 in the category of affine algebraic supergroups in the sense of [22].

\section{Homogeneous supermanifolds}

Suppose that a closed Lie subsupergroup $\left(H, \mathcal{O}_{H}\right)$ of $\left(G, \mathcal{O}_{G}\right)$ (this means that the Lie subgroup $H$ is closed in $G$ ) is given. Consider the corresponding coset superspace $\left(G / H, \mathcal{O}_{G / H}\right)$ (see $\left.[4,6]\right)$. Denote by $\mu_{G \times H}$ the composition of the morphisms

$$
\left(G, \mathcal{O}_{G}\right) \times\left(H, \mathcal{O}_{H}\right) \hookrightarrow\left(G, \mathcal{O}_{G}\right) \times\left(G, \mathcal{O}_{G}\right) \stackrel{\mu}{\rightarrow}\left(G, \mathcal{O}_{G}\right)
$$

by $\operatorname{pr}_{1}:\left(G, \mathcal{O}_{G}\right) \times\left(H, \mathcal{O}_{H}\right) \rightarrow\left(G, \mathcal{O}_{G}\right)$ the projection onto the first factor and by $\pi$ the natural mapping $G \rightarrow G / H, g \mapsto g H$. Let us take $U \subset G / H$ open. Then

$$
\mathcal{O}_{G / H}(U)=\left\{f \in \mathcal{O}_{G}\left(\pi^{-1}(U)\right) \mid\left(\mu_{G \times H}\right)^{*}(f)=\operatorname{pr}_{1}^{*}(f)\right\} .
$$


Denote by $\nu:\left(G, \mathcal{O}_{G}\right) \times\left(G / H, \mathcal{O}_{G / H}\right) \rightarrow\left(G / H, \mathcal{O}_{G / H}\right)$ the natural supergroup action. It is given by $\nu^{*}(f)=\mu^{*}(f)$, where $f \in \mathcal{O}_{G / H}(U) \subset$ $\mathcal{O}_{G}\left(\pi^{-1}(U)\right)$.

A Harish-Chandra pair $(H, \mathfrak{h})$ is called a Harish-Chandra subpair of a Harish-Chandra pair $(G, \mathfrak{g})$ if $H$ is a Lie subgroup of $G$ and $\mathfrak{h}$ is a Lie subsuperalgebra of $\mathfrak{g}$, s.t. $\mathfrak{h}_{\overline{0}}=$ Lie $H$ and $\alpha_{H}=\alpha_{G} \mid H$. There is a correspondence between Harish-Chandra subpairs of $(G, \mathfrak{g})$ and Lie subsupergroups of $\left(G, \mathcal{O}_{G}\right)$. (The Lie supergroup $\left(G, \mathcal{O}_{G}\right)$ corresponds to the Harish-Chandra pair $(G, \mathfrak{g})$.) More precisely, let $\varphi:\left(H, \mathcal{O}_{H}\right) \rightarrow\left(G, \mathcal{O}_{G}\right)$ be a Lie subsupergroup. Then the corresponding Harish-Chandra pair $(H, \mathfrak{h})$ is a HarishChandra subpair, because $H \subset G$ is a Lie subgroup and $\mathfrak{h}=(\mathrm{d} \varphi)_{e} \subset \mathfrak{g}$ is a Lie subalgebra, s.t. $\mathfrak{h}_{\overline{0}}=$ Lie $H$ and $\alpha_{H}=\alpha_{G} \mid H$. Further, let $\varphi$ : $\left(H, \mathcal{O}_{H}\right) \rightarrow\left(G, \mathcal{O}_{G}\right)$ and $\varphi^{\prime}:\left(H^{\prime}, \mathcal{O}_{H^{\prime}}\right) \rightarrow\left(G, \mathcal{O}_{G}\right)$ be two Lie subsupergroups which determine the same Harish-Chandra pair $(H, \mathfrak{h})$. We claim that there is an isomorphism of Lie supergroups $\psi:\left(H, \mathcal{O}_{H}\right) \rightarrow\left(H^{\prime}, \mathcal{O}_{H^{\prime}}\right)$, such that $\varphi^{\prime} \circ \psi=\varphi$. As we have seen above, any homomorphism of Lie supergroups is determined by its underlying map and its differential at the point $e$. To define $\psi$, we put $\psi_{\text {red }}=\mathrm{id}: H \rightarrow H^{\prime}$ (note that $H=H^{\prime}$ ) and $(\mathrm{d} \psi)_{e}=\left(\mathrm{d} \varphi^{\prime}\right)_{e}^{-1} \circ(\mathrm{d} \varphi)_{e}$.

Conversely, let $(H, \mathfrak{h})$ be a Harish-Chandra subpair of $(G, \mathfrak{g})$. Then we get the Lie supergroup $\left(H, \mathcal{O}_{H}\right)$ using the construction from 2.1. There is a natural homomorphism $\varphi:\left(H, \mathcal{O}_{H}\right) \rightarrow\left(G, \mathcal{O}_{G}\right)$, where $\varphi_{\text {red }}: H \rightarrow G$ is the inclusion and $\varphi^{*}: \mathcal{O}_{G} \rightarrow \mathcal{O}_{H}$ is given by

$$
\varphi^{*}(f)(X)(h)=f(X)\left(\varphi_{\text {red }}(h)\right), \quad X \in \mathfrak{U}(\mathfrak{h}) \subset \mathfrak{U}(\mathfrak{g}), \quad h \in H .
$$

Clearly, the Harish-Chandra subpair which corresponds to the Lie subsupergroup $\left(H, \mathcal{O}_{H}\right)$ coincides with $(H, \mathfrak{h})$.

Let $\nu:\left(G, \mathcal{O}_{G}\right) \times\left(M, \mathcal{O}_{M}\right) \rightarrow\left(M, \mathcal{O}_{M}\right)$ be a transitive action. Denote by $\nu_{x}$, where $x \in M$, the composition of the morphisms

$$
\left(G, \mathcal{O}_{G}\right)=\left(G, \mathcal{O}_{G}\right) \times(x, \mathbb{K}) \stackrel{\text { id } \times \delta_{x}}{\longrightarrow}\left(G, \mathcal{O}_{G}\right) \times\left(M, \mathcal{O}_{M}\right) \stackrel{\nu}{\rightarrow}\left(M, \mathcal{O}_{M}\right) .
$$

Lemma 4. We have $\mathrm{ev}_{x} \circ \bar{\nu}(X)=\left(\mathrm{d} \nu_{x}\right)_{e}\left(X_{e}\right), X \in \mathfrak{g}$.

Proof. By definition we get

$$
\mathrm{ev}_{x}(\bar{\nu}(X))(f)=[\bar{\nu}(X)(f)]_{\mathrm{red}}(x), \quad\left(\mathrm{d} \nu_{x}\right)_{e}\left(X_{e}\right)(f)=X_{e} \circ \nu_{x}^{*}(f)
$$

for $f \in\left(\mathcal{O}_{M}\right)_{x}$. Let $\delta_{x}(h):=(h)_{\text {red }}(x), h \in \mathcal{O}_{M}$. Then,

$$
\begin{gathered}
\operatorname{ev}_{x}(\bar{\nu}(X))(f)=\left[\left(X_{e} \otimes \mathrm{id}\right) \circ \nu^{*}(f)\right]_{\mathrm{red}}(x)=\left(X_{e} \otimes \delta_{x}\right) \circ \nu^{*}(f)= \\
X_{e} \circ\left(\mathrm{id} \otimes \delta_{x}\right) \circ \nu^{*}(f)=X_{e} \circ \nu_{x}^{*}(f)=\left(\mathrm{d} \nu_{x}\right)_{e}\left(X_{e}\right)(f) .
\end{gathered}
$$


Remark. By the axioms of an action we have $\nu_{x}=\nu_{g x} \circ r_{g^{-1}}$ for all $g \in G$. Using Lemma 4 we get that a supermanifold $\left(M, \mathcal{O}_{M}\right)$ is $\left(G, \mathcal{O}_{G}\right)$-homogeneous if and only if $\nu_{\text {red }}$ is a transitive action of $G$ on $M$ and $\left(\mathrm{d} \nu_{x}\right)_{e}$ is surjective for some $x \in M$.

As in [6], we can define the stationary subsupergroup $\left(G_{x}, \mathcal{O}_{G_{x}}\right)$ of the point $x$ in the following way. Consider the Harish-Chandra subpair $\left(G_{x}, \mathfrak{g}_{x}\right)$ of $(G, \mathfrak{g})$, where $G_{x}$ is the stabilizer of $x$ and $\mathfrak{g}_{x}=\operatorname{Ker}\left(\mathrm{d} \nu_{x}\right)_{e}$. A subsupergroup $\left(G_{x}, \mathcal{O}_{G_{x}}\right)$ is called the stabilizer of $x$, if it determines $\left(G_{x}, \mathfrak{g}_{x}\right)$. Further, assume that the action $\nu$ is transitive. In this case in [21] another definition of the stabilizer of $x$ was given. It is easy to prove that these two definitions are equivalent. Moreover, $\left(M, \mathcal{O}_{M}\right) \simeq\left(G / G_{x}, \mathcal{O}_{G / G_{x}}\right)$ and hence $\operatorname{dim}\left(M, \mathcal{O}_{M}\right)=$ $\operatorname{dim}\left(G, \mathcal{O}_{G}\right)-\operatorname{dim}\left(G_{x}, \mathcal{O}_{G_{x}}\right)($ see, $[6,21])$.

\section{Homogeneous split supermanifolds}

In this section we will consider only complex supermanifolds. Note that all real supermanifolds are split.

Let us introduce a new category SSM (split supermanifolds). We put

$$
\mathrm{Ob} \mathrm{SSM}=\left\{\left(M, \wedge \mathcal{E}_{M}\right) \mid \mathcal{E}_{M} \text { is a locally free sheaf on } M\right\} .
$$

Equivalently, we can say that Ob SSM consists of all split supermanifolds $\left(M, \mathcal{O}_{M}\right)$ with a fixed isomorphism $\mathcal{O}_{M} \simeq \bigwedge \mathcal{E}_{M}$ for a certain locally free sheaf $\mathcal{E}_{M}$ on $M$. Note that $\mathcal{O}_{M}$ is naturally $\mathbb{Z}$-graded by $\left(\mathcal{O}_{M}\right)_{p} \simeq \bigwedge^{p} \mathcal{E}_{M}$. All the time we will consider this $\mathbb{Z}$-grading. Further, if $X, Y \in \mathrm{Ob}$ SSM we put

$$
\begin{aligned}
\operatorname{Hom}(X, Y)= & \text { all morphisms of } X \text { to } Y, \\
& \text { preserving the } \mathbb{Z} \text {-gradings. }
\end{aligned}
$$

As in the category of supermanifolds, we can define in SSM a group object (split Lie supergroup), an action of a split Lie supergroup on a split supermanifold (split action) and a homogeneous split supermanifold. More precisely, we get these notions if we consider in the definitions 1, 2, 3 morphisms and objects from SSM.

Let $\left(M, \bigwedge \mathcal{E}_{M}\right)$ and $\left(N, \bigwedge \mathcal{E}_{N}\right)$ be two split supermanifolds, where $\mathcal{E}_{M}$ and $\mathcal{E}_{N}$ are the sheaves of sections of vector bundles $\mathbf{E}_{M}$ and $\mathbf{E}_{N}$ respectively. The direct product in the category SSM is defined by:

$$
\left(M, \bigwedge \mathcal{E}_{M}\right) \times\left(N, \bigwedge \mathcal{E}_{N}\right):=\left(M \times N, \bigwedge\left(\mathcal{E}_{M} \oplus \mathcal{E}_{N}\right)\right)
$$


Here the fixed $\mathbb{Z}$-grading is given by

$$
\bigwedge^{p}\left(\mathcal{E}_{M} \oplus \mathcal{E}_{N}\right)=\bigoplus_{t+s=p} \bigwedge^{r} \mathcal{E}_{M} \otimes \bigwedge^{s} \mathcal{E}_{N}
$$

It is easy to see that this definition agrees with the definition of the direct product in the category of supermanifolds, see [9].

There is a functor, say gr, from the category of supermanifolds to the category of split supermanifolds. Let us briefly describe this construction (see, e.g., $[12,15])$. Let $\left(M, \mathcal{O}_{M}\right)$ be a supermanifold. As above denote by $\mathcal{J}_{M} \subset \mathcal{O}_{M}$ the subsheaf of ideals generated by odd elements of $\mathcal{O}_{M}$. Then by definition $\operatorname{gr}\left(M, \mathcal{O}_{M}\right)$ is equal to the split supermanifold $\left(M, \operatorname{gr} \mathcal{O}_{M}\right)$, where

$$
\operatorname{gr} \mathcal{O}_{M}=\bigoplus_{p \geq 0}\left(\operatorname{gr} \mathcal{O}_{M}\right)_{p}, \quad \mathcal{J}_{M}^{0}:=\mathcal{O}_{M}, \quad\left(\operatorname{gr} \mathcal{O}_{M}\right)_{p}=\mathcal{J}_{M}^{p} / \mathcal{J}_{M}^{p+1}
$$

In this case $\left(\operatorname{gr} \mathcal{O}_{M}\right)_{1}$ is a locally free sheaf and there is a natural isomorphism of $\operatorname{gr} \mathcal{O}_{M}$ onto $\bigwedge\left(\operatorname{gr} \mathcal{O}_{M}\right)_{1}$. If $\psi=\left(\psi_{\text {red }}, \psi^{*}\right):\left(M, \mathcal{O}_{M}\right) \rightarrow\left(N, \mathcal{O}_{N}\right)$ is a morphism, then $\operatorname{gr}(\psi)=\left(\psi_{\text {red }}, \operatorname{gr}\left(\psi^{*}\right)\right)$, where $\operatorname{gr}\left(\psi^{*}\right): \operatorname{gr} \mathcal{O}_{N} \rightarrow \operatorname{gr} \mathcal{O}_{M}$ is defined by

$$
\operatorname{gr}\left(\psi^{*}\right)\left(f+\mathcal{J}_{N}^{p}\right):=\psi^{*}(f)+\mathcal{J}_{M}^{p} \text { for } f \in\left(\mathcal{J}_{N}\right)^{p-1} .
$$

Recall that by definition every morphism $\psi$ of supermanifolds is even and as consequence sends $\mathcal{J}_{N}^{p}$ into $\mathcal{J}_{M}^{p}$.

Let $\left(G, \mathcal{O}_{G}\right)$ be a Lie supergroup with the group morphisms $\mu, \iota$ and $\varepsilon$. Then it is easy to see that $\operatorname{gr}\left(G, \mathcal{O}_{G}\right)$ is a split Lie supergroup with the group morphisms $\operatorname{gr}(\mu), \operatorname{gr}(\iota)$ and $\operatorname{gr}(\varepsilon)$. Similarly, an action $\nu:\left(G, \mathcal{O}_{G}\right) \times$ $\left(M, \mathcal{O}_{M}\right) \rightarrow\left(M, \mathcal{O}_{M}\right)$ gives rise to the action $\operatorname{gr}(\nu): \operatorname{gr}\left(G, \mathcal{O}_{G}\right) \times \operatorname{gr}\left(M, \mathcal{O}_{M}\right) \rightarrow$ $\operatorname{gr}\left(M, \mathcal{O}_{M}\right)$.

Obviously a split Lie supergroup is a Lie supergroup. Furthermore, the following result holds:

Proposition 4. A Lie supergroup $\left(G, \mathcal{O}_{G}\right)$ with the Lie superalgebra $\mathfrak{g}$ possesses a structure of a split Lie supergroup if and only if $\left[\mathfrak{g}_{\overline{1}}, \mathfrak{g}_{\overline{1}}\right]=0$. Any Lie subsupergroup of a split Lie supergroup possesses a structure of a split Lie supergroup.

Proof. Let $\left(G, \mathcal{O}_{G}\right)$ be a split Lie supergroup, $\mathcal{O}_{G}=\bigoplus_{p}\left(\mathcal{O}_{G}\right)_{p}$ the fixed $\mathbb{Z}$ grading and $\mu$ the multiplication morphism. Let us prove that $\left[\mathfrak{g}_{\overline{1}}, \mathfrak{g}_{\overline{1}}\right]=\{0\}$. It is enough to check that $[X, Y](f)=0$ for $f \in\left(\mathcal{O}_{G}\right)_{0}$ and $f \in\left(\mathcal{O}_{G}\right)_{1}$, where $X, Y \in \mathfrak{g}_{1}$. By $(2)$ we get

$$
\begin{gathered}
{[X, Y](f)=(X \otimes \mathrm{id}) \circ \mu^{*} \circ(Y \otimes \mathrm{id}) \circ \mu^{*}(f)+} \\
(Y \otimes \mathrm{id}) \circ \mu^{*} \circ(X \otimes \mathrm{id}) \circ \mu^{*}(f)= \\
-((Y \otimes X \otimes \mathrm{id})+(X \otimes Y \otimes \mathrm{id})) \circ\left(\mu^{2}\right)^{*}(f),
\end{gathered}
$$


where $\mu^{2}$ is the multiplication morphism of three copies of $\left(G, \mathcal{O}_{G}\right)$. Note that by definition of a split Lie supergroup $\left(\mu^{2}\right)^{*}(f) \in\left(\mathcal{O}_{G \times G \times G}\right)_{0}$, if $f \in\left(\mathcal{O}_{G}\right)_{0}$ and $\left(\mu^{2}\right)^{*}(f) \in\left(\mathcal{O}_{G \times G \times G}\right)_{1}$, if $f \in\left(\mathcal{O}_{G}\right)_{1}$. It follows that

$$
(X \otimes Y \otimes \mathrm{id})\left(\left(\mu^{2}\right)^{*}(f)\right)=(Y \otimes X \otimes \mathrm{id})\left(\left(\mu^{2}\right)^{*}(f)\right)=0 .
$$

Conversely, let $\left(G, \mathcal{O}_{G}\right)$ be a Lie supergroup and $\left[\mathfrak{g}_{\overline{1}}, \mathfrak{g}_{\overline{1}}\right]=0$. As we have seen above, the sheaf $\mathcal{O}_{G}=\operatorname{Hom}_{\mathfrak{U}\left(\mathfrak{g}_{\overline{0}}\right)}\left(\mathfrak{U}(\mathfrak{g}), \mathcal{F}_{G}\right)$ is a $\mathbb{Z}$-graded sheaf. Recall that the $\mathbb{Z}$-grading is induced by the mapping $\mathcal{O}_{G} \rightarrow \operatorname{Hom}\left(\bigwedge\left(\mathfrak{g}_{1}\right), \mathcal{F}_{G}\right)$, $f \mapsto f \circ \gamma$, where $\gamma$ is defined by (4). More precisely,

$$
f \in\left(\mathcal{O}_{G}\right)_{p} \Leftrightarrow f \circ \gamma\left(X_{1} \wedge \cdots \wedge X_{r}\right)=0 \text { for } r \neq p, X_{i} \in \mathfrak{g}_{\overline{1}} .
$$

It follows that any Lie supergroup is contained in Ob SSM. We want to prove that the structure morphisms $(7)$ of $\left(G, \mathcal{O}_{G}\right)$ preserve this $\mathbb{Z}$-grading. Let us check that $\mu^{*}\left(\left(\mathcal{O}_{G}\right)_{p}\right) \subset\left(\mathcal{O}_{G \times G}\right)_{p}, p \geq 0$. Further, if $\left[\mathfrak{g}_{\overline{1}}, \mathfrak{g}_{\overline{1}}\right]=0$, then $\gamma$ is a homomorphism of algebras (not only of coalgebras), see (4). It follows that

$$
f \in\left(\mathcal{O}_{G}\right)_{p} \Leftrightarrow f\left(X_{1} \cdots X_{r}\right)=0 \text { for } r \neq p, X_{i} \in \mathfrak{g}_{\overline{1}} .
$$

Let $X_{1}, \ldots, X_{r}$ be from the first copy of $\mathfrak{g}_{\overline{1}}$ and $Y_{1}, \ldots, Y_{q}$ be from the second one, $r+q \neq p, g, h \in G$ and $f \in\left(\mathcal{O}_{G}\right)_{p}$, then

$$
\mu^{*}(f)\left(X_{1} \cdots X_{r} \cdot Y_{1} \cdots Y_{q}\right)(g, h)=f\left(X_{1} \cdots X_{r} \cdot \alpha_{G}(g)\left(Y_{1} \cdots Y_{q}\right)\right)(g h)=0
$$

This implies that $\mu^{*}(f) \in\left(\mathcal{O}_{G \times G}\right)_{p}$. For the inversion morphism the proof is similar.

The second assertion is obvious. Indeed, if $\left(H, \mathcal{O}_{H}\right) \subset\left(G, \mathcal{O}_{G}\right)$ is a Lie subsupergroup, $\mathfrak{h}=\operatorname{Lie}\left(H, \mathcal{O}_{H}\right)$, then $\mathfrak{h} \subset \mathfrak{g}$ is a subsuperalgebra. Hence, $\left[\mathfrak{h}_{\overline{1}}, \mathfrak{h}_{\overline{1}}\right]=0$.

Corollary. A split Lie supergroup is a semi-direct product of a usual Lie group $G$ and the (unique) connected supergroup of purely odd dimension.

Lemma 5. Let $\nu:\left(G, \mathcal{O}_{G}\right) \times\left(M, \mathcal{O}_{M}\right) \rightarrow\left(M, \mathcal{O}_{M}\right)$ be a transitive action of a Lie supergroup $\left(G, \mathcal{O}_{G}\right)$ on a supermanifold $\left(M, \mathcal{O}_{M}\right)$, then the action

$$
\operatorname{gr} \nu:\left(G, \operatorname{gr} \mathcal{O}_{G}\right) \times\left(M, \operatorname{gr} \mathcal{O}_{M}\right) \rightarrow\left(M, \operatorname{gr} \mathcal{O}_{M}\right)
$$

is also transitive. In particular, if $\left(M, \mathcal{O}_{M}\right)$ is split and homogeneous, then it always admits a transitive split action.

Proof. Since gr $\nu_{\text {red }}=\nu_{\text {red }}$ it is enough to show that $\mathrm{d}\left(\operatorname{gr} \nu_{x}\right)_{e}$ is surjective for some $x \in M$ (see Remark after Lemma 4). Since $\mathrm{d}\left(\operatorname{gr} \nu_{x}\right)_{e}=\mathrm{d}\left(\nu_{x}\right)_{e}$, 
the proof is complete. The second assertion follows from the isomorphism $\left(M, \mathcal{O}_{M}\right) \simeq\left(M, \operatorname{gr} \mathcal{O}_{M}\right)$

Let $H$ be a closed Lie subgroup of a Lie group $G, E$ a complex vector space and $\theta: H \rightarrow \mathrm{GL}(E)$ be a holomorphic representation. Denote by $\mathcal{E}^{\theta}$ the sheaf of sections of the homogeneous vector bundle $\mathbf{E}^{\theta}$ which corresponds to $\theta$, i.e., the quotient space of the direct product $G \times E$ by the following action of $H$ :

$$
(g, v) \stackrel{h}{\mapsto}\left(g h^{-1}, \theta(h) v\right), \quad g \in G, h \in H, v \in E .
$$

Furthermore, let $\pi: G \rightarrow G / H$ be the natural projection and $U \subset G / H$ open. There is an injective homomorphism of sheaves $\Phi_{\theta}: \mathcal{E}^{\theta} \rightarrow \pi_{*}\left(\mathcal{F}_{G} \otimes E\right)$ given by

$$
\begin{aligned}
\mathcal{E}^{\theta}(U) \ni s & \mapsto f_{s} \in \mathcal{F}_{G}\left(\pi^{-1}(U)\right) \otimes E \\
f_{s}(g) & :=g^{-1} s(g H), g \in G .
\end{aligned}
$$

It is well known that

$$
\Phi_{\theta}\left(\mathcal{E}^{\theta}(U)\right)=\left\{f \in \mathcal{F}_{G}\left(\pi^{-1}(U)\right) \otimes E \mid \theta(h) f(g h)=f(g), g \in G, h \in H\right\} .
$$

Note that $\bigwedge \mathcal{E}^{\theta}=\mathcal{E}^{\wedge \theta}$ is also a homogeneous bundle. An easy computation shows that $\Phi_{\wedge \theta}: \bigwedge \mathcal{E}^{\theta} \rightarrow \pi_{*}\left(\mathcal{F}_{G} \otimes \bigwedge E\right)$ is a homomorphism of the sheaves of superalgebras.

Let $V$ be a vector space. Our aim is now to describe the isomorphism of sheaves of superalgebras

$$
\Psi_{V}: \mathcal{F}_{G} \otimes \bigwedge V^{*} \rightarrow \operatorname{Hom}\left(\bigwedge V, \mathcal{F}_{G}\right)
$$

mentioned in 2.1. Let $\left(\xi_{i}\right)$ be a basis of $V,\left(\xi_{i}^{*}\right)$ the dual basis of $V^{*}, h \in \mathcal{F}_{G}$ and $i_{1}<\cdots<i_{k}, j_{1}<\cdots<j_{r}$. Then

$$
\begin{aligned}
& \Psi_{V}\left(h \xi_{i_{1}}^{*} \wedge \cdots \wedge \xi_{i_{k}}^{*}\right)=(-1)^{k(k-1) / 2} h f^{\xi_{i_{1}} \wedge \cdots \wedge \xi_{i_{k}}} \text {, where }
\end{aligned}
$$

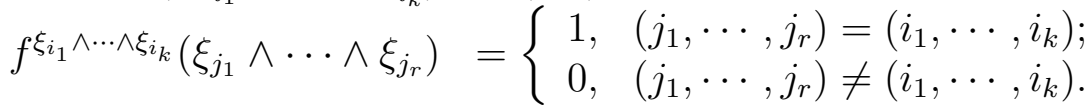

The direct computation shows that it is a homomorphism of the sheaves of superalgebras.

Proposition 5. Let $\left(G, \mathcal{O}_{G}\right)$ be a split Lie supergroup, $\left(H, \mathcal{O}_{H}\right) \subset\left(G, \mathcal{O}_{G}\right)$ a closed Lie subsupergroup and $\left(M, \mathcal{O}_{M}\right):=\left(G / H, \mathcal{O}_{G / H}\right)$. The $\mathbb{Z}$-grading $\mathcal{O}_{G}=\bigoplus_{p \geq 0}\left(\mathcal{O}_{G}\right)_{p}$, where $\left(\mathcal{O}_{G}\right)_{p}$ is determined by (18), induces the $\mathbb{Z}$-grading $\left(\mathcal{O}_{M}\right)_{p}$ on the subsheaf $\mathcal{O}_{M} \subset \mathcal{O}_{G}$. Moreover, $\left(\mathcal{O}_{M}\right)_{1}$ is a locally free sheaf and $\left(\mathcal{O}_{M}\right)_{p}=\bigwedge^{p}\left(\mathcal{O}_{M}\right)_{1}$. 
In particular, the coset supermanifold $\left(M, \mathcal{O}_{M}\right)$ is split and the natural action of the Lie supergroup $\left(G, \mathcal{O}_{G}\right)$ on $\left(M, \mathcal{O}_{M}\right)$ is split.

Proof. The sheaf $\mathcal{O}_{M}$ was defined by (17). The supergroup $\left(G, \mathcal{O}_{G}\right)$ is a split Lie supergroup, hence $\left(\mu_{G \times H}\right)^{*}\left(\left(\mathcal{O}_{G}\right)_{p}\right) \subset\left(\mathcal{O}_{G \times H}\right)_{p}$. Furthermore, it is easy to see that $\operatorname{pr}_{1}^{*}\left(\left(\mathcal{O}_{G}\right)_{p}\right) \subset\left(\mathcal{O}_{G \times H}\right)_{p}$. Hence the sheaf $\mathcal{O}_{M}$ is $\mathbb{Z}$-graded by the subsheaves

$$
\left(\mathcal{O}_{M}\right)_{p}=\left\{f \in\left(\mathcal{O}_{G}\right)_{p} \mid\left(\mu_{G \times H}\right)^{*}(f)=\operatorname{pr}_{1}^{*}(f)\right\} .
$$

Consider the representation $\psi: H \rightarrow G L\left(\left(\mathfrak{g}_{\overline{1}} / \mathfrak{h}_{\overline{1}}\right)^{*}\right)$ defined by $[\psi(h)(v)]\left(X+\mathfrak{h}_{\overline{1}}\right)=v\left(\operatorname{Ad}_{G}\left(h^{-1}\right)(X)+\mathfrak{h}_{\overline{1}}\right)$ for $h \in H, v \in\left(\mathfrak{g}_{\overline{1}} / \mathfrak{h}_{\overline{1}}\right)^{*}, \quad X \in \mathfrak{g}_{\overline{1}}$.

Our goal now is to show that $\mathcal{O}_{M} \simeq \bigwedge \mathcal{E}^{\psi}$ as sheaves of $\mathbb{Z}$-graded algebras. Denote by $\Gamma$ the isomorphism of sheaves $\mathcal{O}_{G}=\widehat{\mathcal{O}}_{G} \rightarrow \operatorname{Hom}\left(\bigwedge\left(\mathfrak{g}_{\overline{1}}\right), \mathcal{F}_{G}\right)$ described in 2.1. We have

$$
\mathcal{O}_{M} \subset \widehat{\mathcal{O}}_{G} \stackrel{\Gamma}{\longrightarrow} \operatorname{Hom}\left(\bigwedge\left(\mathfrak{g}_{\overline{1}}\right), \mathcal{F}_{G}\right) .
$$

By definition $f \in\left(\mathcal{O}_{M}\right)_{p}$ if and only if $f \in\left(\widehat{\mathcal{O}}_{G}\right)_{p}$ and $\left(\mu_{G \times H}\right)^{*}(f)=\operatorname{pr}_{1}^{*}(f)$. Using (7) and (12) we can write the last condition in the following form:

$$
f\left(X^{r} \cdot \operatorname{Ad}_{G}(g)\left(Y^{q}\right)\right)(g h)= \begin{cases}0, & \text { if } q \neq 0 ; \\ f\left(X^{r}\right)(g), & \text { if } q=0\end{cases}
$$

for all $g \in G, h \in H$, where $X^{r}=X_{1} \cdots X_{r}, X_{i} \in \mathfrak{g}, Y^{q}=Y_{1} \cdots Y_{q}, Y_{j} \in \mathfrak{h}=$ $\operatorname{Lie}\left(H, \mathcal{O}_{H}\right), r+q=p$. The supergroup $\left(G, \mathcal{O}_{G}\right)$ is a split Lie supergroup, it follows by Proposition 4 that $\left[\mathfrak{g}_{\overline{1}}, \mathfrak{g}_{\overline{1}}\right]=0$. As we mentioned above, in this case the mapping (4) is an injective homomorphism of superalgebras (not only of cosuperalgebras). Hence $s \in \Gamma\left(\left(\mathcal{O}_{M}\right)_{p}\right)$ if and only if $s \in \operatorname{Hom}\left(\bigwedge^{p}\left(\mathfrak{g}_{\overline{1}}\right), \mathcal{F}_{G}\right)$ and the following condition holds:

$$
s\left(X^{r} \wedge \operatorname{Ad}_{G}(g)\left(Y^{q}\right)\right)(g h)= \begin{cases}0, & \text { if } q \neq 0 \\ s\left(X^{r}\right)(g), & \text { if } q=0\end{cases}
$$

for all $g \in G, h \in H, X^{r}=X_{1} \wedge \cdots \wedge X_{r} \in \wedge^{r}\left(\mathfrak{g}_{\overline{1}}\right), Y^{q}=Y_{1} \wedge \cdots \wedge Y_{q} \in \bigwedge^{q}\left(\mathfrak{h}_{\overline{1}}\right)$, $r+q=p$.

We may regard the sheaf $\operatorname{Hom}\left(\bigwedge\left(\mathfrak{g}_{\overline{1}} / \mathfrak{h}_{\overline{1}}\right), \mathcal{F}_{G}\right)$ as a sheaf of superalgebras. The multiplication is defined as in 2.1. Define the injective homomorphism of sheaves of superalgebras in the following way:

$$
\begin{gathered}
\Upsilon: \operatorname{Hom}\left(\bigwedge\left(\mathfrak{g}_{\overline{1}} / \mathfrak{h}_{\overline{1}}\right), \mathcal{F}_{G}\right) \longrightarrow \operatorname{Hom}\left(\bigwedge\left(\mathfrak{g}_{\overline{1}}\right), \mathcal{F}_{G}\right), \\
\Upsilon(f)\left(\operatorname{Ad}_{G}(g)(X)\right)(g)=f(\bar{X})(g),
\end{gathered}
$$


where $X \in \bigwedge\left(\mathfrak{g}_{\overline{1}}\right), \bar{X}$ is the image of $X$ by the natural homomorphism $\bigwedge\left(\mathfrak{g}_{\overline{1}}\right) \rightarrow \bigwedge\left(\mathfrak{g}_{\overline{1}} / \mathfrak{h}_{\overline{1}}\right), g \in G$.

Consider the composition of the injective homomorphisms of sheaves of superalgebras

$$
\bigwedge \mathcal{E}^{\psi} \stackrel{\Phi_{\curlywedge \psi}}{\longrightarrow} \mathcal{F}_{G} \otimes \bigwedge\left(\mathfrak{g}_{\overline{1}} / \mathfrak{h}_{\overline{1}}\right)^{*} \stackrel{\Psi}{\stackrel{\Psi_{\left(\mathfrak{g}_{\overline{1}} / \mathfrak{h}_{\overline{1}}\right)^{*}}}{\longrightarrow}} \operatorname{Hom}\left(\bigwedge\left(\mathfrak{g}_{\overline{1}} / \mathfrak{h}_{\overline{1}}\right), \quad \begin{array}{l}
\left.\mathcal{F}_{G}\right) \\
\stackrel{\Upsilon}{\longrightarrow} \operatorname{Hom}\left(\bigwedge\left(\mathfrak{g}_{\overline{1}}\right), \mathcal{F}_{G}\right) .
\end{array}\right.
$$

Our goal now is to show that

$$
\Gamma\left(\left(\mathcal{O}_{M}\right)_{p}\right)=\Upsilon \circ \Psi_{\bigwedge\left(\mathfrak{g}_{\overline{1}} / \mathfrak{h}_{\overline{1}}\right)^{*}} \circ \Phi_{\wedge \psi}\left(\bigwedge^{p} \mathcal{E}^{\psi}\right), p \geq 0
$$

This will imply our assertion.

Note that $f \in \Psi_{\bigwedge\left(\mathfrak{g}_{\overline{1}} / \mathfrak{h}_{\overline{1}}\right)^{*}} \circ \Phi_{\wedge \psi}\left(\bigwedge^{p} \mathcal{E}^{\psi}\right)$ if and only if $f \in \operatorname{Hom}\left(\bigwedge^{p}\left(\mathfrak{g}_{\overline{1}} / \mathfrak{h}_{\overline{1}}\right), \mathcal{F}_{G}\right)$ and the following condition holds:

$$
f\left(\operatorname{Ad}_{G}\left(h^{-1}\right)(\bar{X})\right)(g h)=f(\bar{X})(g),
$$

where $X \in \bigwedge^{p}\left(\mathfrak{g}_{\overline{1}}\right), g \in G, h \in H$. Further, $s \in \Upsilon \circ \Psi_{\bigwedge\left(\mathfrak{g}_{\overline{1}} / \mathfrak{h}_{\overline{1}}\right)^{*}} \circ \Phi_{\wedge \psi}\left(\bigwedge^{p} \mathcal{E}^{\psi}\right)$ if and only if $s \in \operatorname{Hom}\left(\bigwedge^{p}\left(\mathfrak{g}_{1}\right), \mathcal{F}_{G}\right)$ and the following condition holds:

$$
s\left(\operatorname{Ad}_{G}(g)(X)\right)(g h)= \begin{cases}0, & \text { if } \bar{X}=\overline{0} \\ s\left(\operatorname{Ad}_{G}(g)(X)\right)(g), & \text { if } \bar{X} \neq \overline{0}\end{cases}
$$

The conditions (20) and (21) are equivalent.

To complete the proof, we recall that the action $\nu:\left(G, \mathcal{O}_{G}\right) \times\left(M, \mathcal{O}_{M}\right) \rightarrow$ $\left(M, \mathcal{O}_{M}\right)$ is defined by $\nu^{*}(f)=\mu^{*}(f), f \in \mathcal{O}_{M} \subset \mathcal{O}_{G}$, and the map $\mu^{*}$ and the inclusion $\mathcal{O}_{G \times M} \hookrightarrow \mathcal{O}_{G \times G}$ preserve the chosen $\mathbb{Z}$-gradings.

Let us formulate the general result concerning a complex homogeneous split supermanifold.

Theorem 2. Let $\left(G, \mathcal{O}_{G}\right)$ be a complex Lie supergroup with the Lie superalgebra $\mathfrak{g}=\mathfrak{g}_{\overline{0}} \oplus \mathfrak{g}_{\overline{1}}$. If $\left[\mathfrak{g}_{\overline{1}}, \mathfrak{g}_{\overline{1}}\right]=0$ then all $\left(G, \mathcal{O}_{G}\right)$-homogeneous supermanifolds $\left(M, \mathcal{O}_{M}\right)$ are split supermanifolds. Moreover, the sheaf $\mathcal{O}_{M}$ is isomorphic to $\bigwedge \mathcal{E}^{\psi}$, where $\mathcal{E}^{\psi}$ is the sheaf of sections of the homogeneous vector bundle $\mathbf{E}^{\psi}$, which corresponds to the representation $\psi: H \rightarrow G L\left(\left(\mathfrak{g}_{\overline{1}} / \mathfrak{h}_{\overline{1}}\right)^{*}\right)$ given by

$\psi(h)(v)\left(X+\mathfrak{h}_{\overline{1}}\right):=v\left(\operatorname{Ad}_{G}\left(h^{-1}\right)(X)+\mathfrak{h}_{\overline{1}}\right)$, for $h \in H, X \in \mathfrak{g}_{\overline{1}}, v \in\left(\mathfrak{g}_{\overline{1}} / \mathfrak{h}_{\overline{1}}\right)^{*}$

Conversely, if a complex homogeneous supermanifold $\left(M, \mathcal{O}_{M}\right)$ is split then there is a Lie supergroup $\left(G, \mathcal{O}_{G}\right)$ with $\left[\mathfrak{g}_{\overline{1}}, \mathfrak{g}_{\overline{1}}\right]=0$, where $\mathfrak{g}=\mathfrak{g}_{\overline{0}} \oplus \mathfrak{g}_{\overline{1}}=$ $\operatorname{Lie}\left(G, \mathcal{O}_{G}\right)$, such that $\left(G, \mathcal{O}_{G}\right)$ acts on $\left(M, \mathcal{O}_{M}\right)$ transitively. 
Proof. The theorem follows from Propositions 4, 5 and Lemma 5.

Let us prove for example that the complex projective superspace $\mathbb{C P}^{1 / 2}$ is split. It is isomorphic to the coset space $\mathrm{GL}_{2 \mid 1}(\mathbb{C}) /\left(P, \mathcal{O}_{P}\right)$, where

$$
\mathrm{GL}_{2 \mid 1}(\mathbb{C})=\left(\begin{array}{ccc}
* & * & \checkmark \\
* & * & \checkmark \\
\checkmark & \checkmark & *
\end{array}\right),\left(P, \mathcal{O}_{P}\right)=\left(\begin{array}{ccc}
* & * & \checkmark \\
0 & * & \checkmark \\
0 & \checkmark & *
\end{array}\right) .
$$

Here $*$ are even coordinates and $\checkmark$ are odd coordinates. It is easy to see that

$$
\mathrm{GL}_{2 \mid 1}(\mathbb{C}) /\left(P, \mathcal{O}_{P}\right) \simeq\left(G^{\prime}, \mathcal{O}_{G^{\prime}}\right) /\left(P^{\prime}, \mathcal{O}_{P^{\prime}}\right),
$$

where

$$
\left(G^{\prime}, \mathcal{O}_{G^{\prime}}\right)=\left(\begin{array}{ccc}
* & * & 0 \\
* & * & 0 \\
\checkmark & \checkmark & *
\end{array}\right),\left(P^{\prime}, \mathcal{O}_{P^{\prime}}\right)=\left(\begin{array}{ccc}
* & * & 0 \\
0 & * & 0 \\
0 & \checkmark & *
\end{array}\right) .
$$

Let $\mathfrak{g}^{\prime}=\operatorname{Lie}\left(G^{\prime}, \mathcal{O}_{G^{\prime}}\right)$. Then

$$
\mathfrak{g}_{1}^{\prime}=\left\{\left(\begin{array}{lll}
0 & 0 & 0 \\
0 & 0 & 0 \\
a & b & 0
\end{array}\right), \quad a, b \in \mathbb{C}\right\} .
$$

We see that $\left[\mathfrak{g}_{1}^{\prime}, \mathfrak{g}_{1}^{\prime}\right]=\{0\}$. By Theorem 2 we get that $\mathbb{C P}^{1 / 2}$ is split.

We close this section by mentioning some results about non-split supermanifolds. The first example of a non-split supermanifold was published in [5]; this is the quadric in the projective superplane $\mathbb{C P}^{2 \mid 2}$. In [10] four series of supermanifolds of flags were constructed corresponding to four series of classical linear Lie superalgebras. In [8] it was proved that all split complex supermanifolds whose reduction is projective algebraic are projective (that is, embeddable in a complex projective superspace.) Penkov and Skornyakov [17] found necessary and sufficient conditions for a supermanifold of flags to be projective. More precisely, they showd that almost all such supermanifolds are not projective. From these two results it follows that supermanifolds of flags are mostly non-split.

In [16] it was proved that the isotropic super-Grassmannian of maximal type $\mathbb{I} \mathbb{G r}_{n|n, s| t}(\mathbb{C})$ associated with an odd bilinear form is non-split whenever $t \geq 1$ and $s \geq 2$. In [15] the complete solution of the problem was given for the isotropic super-Grassmannian of maximal type associated with an even bilinear form. Note that the method of [15] and [16] can be used for all series of flag supermanifolds. 
In [13] the problem of classifying all homogeneous complex supermanifolds whose reduction is the complex Grassmannian $\mathrm{Gr}_{n \mid k}$ was studied. Under the assumption that the odd isotropy representation is irreducible and under certain restrictions on $(n \mid k)$, it was proved that the only non-split supermanifold of this sort is the $\Pi$-symmetric super-Grassmannian constructed by Manin [10].

The problem of classification of non-split supermanifolds having as retract the split supermanifold $(M, \Omega)$, where $\Omega$ is the sheaf of holomorphic forms on a given complex manifold $M$ of dimension $>1$, was studied in [14]. In the case when $M$ is an irreducible compact Hermitian symmetric space, the complete classification of non-split supermanifolds with retract $(M, \Omega)$ was given.

Acknowledgment. The author is grateful to A.L. Onishchik, P. Heinzner, A. T. Huckleberry and anonymous referees for useful comments.

\section{References}

[1] Baguis P., Stavracou T. Normal Lie subsupergroups and non-abelian supercircles. International Journal of Mathematics and Mathematical Sciences Volume 30 (2002), Issue 10, Pages 581-591.

[2] Berezin F.A., Leites D.A. Supermanifolds. Soviet Math. Dokl. 16, 1975, 1218-1222.

[3] Deligne P., Morgan J.W. Notes on supersymmetry (following Joseph Bernstein), Quantum Fields and Strings: A Course for Mathematicians, Vols. 1,2 (Princeton, NJ, 1996/1997), 41-97. American Mathematical Society. Providence, R.I. 1999.

[4] Fioresi R., Lledo M.A., Varadarajan V. S. The super Minkowski and conformal space times, JMP, 48, no. 11, pg. 113505, 2007.

[5] Green P. On holomorphic graded manifolds, Proc. Amer. Math. Soc. 85 (1982), no. 4, 587-590.

[6] Kostant B. Graded manifolds, graded Lie theory, and prequantization. Lecture Notes in Mathematics 570. Berlin e.a.: Springer-Verlag, 1977. P. 177-306.

[7] Koszul J.L. Graded manifolds and graded Lie algebras. Proceeding of the International Meeting on Geometry and Physics (Bologna), Pitagora, 1982, pp 71-84. 
[8] LeBrun C., Poon Y. S., Wells R. O., Jr. Projective embeddings of complex supermanifolds. Comm. Math. Phys. 126 (1990), no. 3, 433452 .

[9] Leites D.A. Introduction to the theory of supermanifolds. Russian Math. Surveys 35 (1980), 1-64.

[10] Manin Yu. I. Gauge field theory and complex geometry. Grundlehren der Mathematischen Wissenschaften, 289, Springer-Verlag, Berlin, 1997.

[11] Molotkov V. Infinite-dimensional $\mathbb{Z}_{2}^{k}$-supermanifolds. International center for theoretical physics, Preprint IC/84/183, 1984, http://ccdb4fs.kek.jp/cgi-bin/img/allpdf?198506284.

[12] Onishchik A.L. Flag supermanifolds, their automorphisms und deformations. The Sophus Lie Memorial conference (Oslo, 1992), 289-302, Scand. Univ. Press, Oslo, 1994.

[13] Onishchik A.L. Homogeneous supermanifolds over Grassmannians, J. Algebra 313 (2007), no. 1, P. 320-342.

[14] Onishchik A.L. Non-split supermanifolds associated with the cotangent bundle. Université de Poitiers, Département de Math., N 109. Poitiers, 1997.

[15] Onishchik A.L., Serov A.A. Vector fields and deformations of isotropic super-Grassmannians of maximal type. Lie Groups and Lie Algebras: E.B. Dynkin's Seminar, AMS Transl. Ser. 2. V. 169. Providence: AMS, 1995. P. 75-90.

[16] Onishchik A.L., Serov A.A. On isotropic super-Grassmannians of maximal type associated with an odd bilinear form. E. Schrödinger Inst. for Math. Physics, Preprint No. 340. Vienna, 1996.

[17] Penkov I.B., Skornyakov I.A. Projectivity and D-affineness of flag supermanifolds, Russ. Math. Surv. 40, (1987) P. 233-234.

[18] Scheunert $M$. The theory of Lie superalgebras. Lectures Notes in Mathematics 716, Springer, Berlin 1979.

[19] Tsalenko M.S., Shulgeifer E.G. Foundations of the theory of categories. Nauka, Moscow 1974 (in Russian).

[20] Varadarajan V. S. Supersymmetry for mathematicians: an introduction, AMS, Courant lecture notes, Vol. 11, 2004. 
[21] Vishnyakova E. G. On the structure of complex homogeneous supermanifolds. arXiv:0811.2581 (November 2008).

[22] Westra D.B. Superrings and supergroups. PhD thesis, Universität Wien, 2009.

Ruhr-Universität Bochum, Universitätssstrasse 150, 44780 Bochum, Germany;

Tver State University, Zhelyabova 33, 170000 Tver, Russia;

E-mail address: VishnyakovaE@googlemail.com 